\title{
Reliability analysis and Response Based Design of a moored FPSO in West Africa
}

\author{
Fontaine E. ${ }^{1,}{ }^{\star}$, Orsero P. ${ }^{2}$, Ledoux Annick ${ }^{3}$, Nerzic R. ${ }^{4}$, Prevosto Marc ${ }^{5}$, Quiniou Valerie 6
}

${ }^{1}$ IFP Energies Nouvelles, Dept Appl Mech, F-92852 Rueil Malmaison, France.

2 Univ Technol Compiegne, F-60203 Compiegne, France.

3 Principia, F-13600 La Ciotat, France.

${ }^{4}$ Actimar, F-29200 Brest, France.

5 IFREMER, Ctr Plouzane, F-29000 Brest, France.

6 Total, F-92078 Paris, France.

* Corresponding author : E. Fontaine, email address : emmanuel fontaine@me.com

\begin{abstract}
:
The present study is an attempt to re-assess the level of reliability of the mooring system of an existing Floating Production Storage and Offloading (FPSO) unit in West Africa. The study made use of field data for the environment including wind, waves and current together with simultaneous measurements of the FPSO offset and of the mooring line tensions. Three different approaches to predict the extreme response are compared. More specifically, the traditional design method is compared with Response Based Design (RBD) and First Order Reliability Method (FORM) analysis associated with Response Surface Models (RSM) of the moored FPSO. The results of this case study allow assessing the level of conservatism that is currently embedded in classical design rules.
\end{abstract}

\section{Highlights}

A reliability analysis of an FPSO mooring system is proposed. Field data including wind, waves and current are used. Models for FPSO offset and the mooring line tensions were benchmarked. The traditional design method is compared with Response Based Design (RBD) and First Order Reliability Method (FORM) analysis associated with Response Surface Models (RSM). D The level of conservatism currently embedded in design rules for moored structures is assessed.

Keywords : Environmental contour, Joint probabilities, Multivariate extremes, I-FORM, FPSO, West Africa, Structural reliability, Response-based design, Inverse reliability method, Response surface 


\section{Introduction}

The Oil and Gas industry extensively uses ship-shaped stucture that are moored on site, also known as Floating Production Storage and Offloading (FPSO) units. These structures as illustrated in Fig. 1 and 2 rely on their mooring system to ensure station keeping so that the FPSO displacements (or offset) remain within a prescribed boundary that can be accomodated by the riser system. The integrity of the mooring system is therefore of critical importance as the failure of one (or more) mooring line(s) may result in catastrophic consequences. Several mooring line failures have been observed all over the world over the last few years, sometimes leading to a complete un-zipping of the mooring system. The observed failure rate as reported in [1] is well above what would be expected according to the design guidance, see e.g. [2] and mooring line failures are expected to continue occuring as the FPSO fleet is aging. Being able to assess the level of reliability of existing mooring systems is therefore of the outmost importance.

The standard approach to design a mooring system consists in computing the response of the moored structure to extreme environmental conditions following the recommended practice of a Class Society and applying factors of safety on the maximum design tensions that have been computed in different configurations such as all mooring lines intact, one or possibly two broken lines. It is worth noting that the values for the Factor of Safety to be applied depend on the loading condition (extreme or survival cases), the chosen standard and the computation method that has been retained to perform the analysis $[3,4,5]$. As a result of using practical design rules with semi-empirical values for the factors of safety, the achieved level of reliability of the mooring system has somewhat been lost in the design process.

The present study is an attempt to re-assess a posteriori the level of reliability of an existing mooring system to estimate the level of conservatism that is currently embeded into the design guidance. The study made use of simultaneous experimental data for the environment (wind, waves and current) and for the FPSO response (offset and mooring line tensions). The studied configuration shown in Fig. 2 comprises the FPSO, a spread moored system with 16 mooring lines grouped in four clusters, three riser towers and two export lines by water depths ranging from 1250 to $1400 \mathrm{~m}$. Results of the classical design methodology as per Recommended Practice [3] were compared to the ones 
from Response-Based-Design (RBD) and First Order Reliability Method (FORM). More information on this study can also be found in $[6,7,8,9]$.

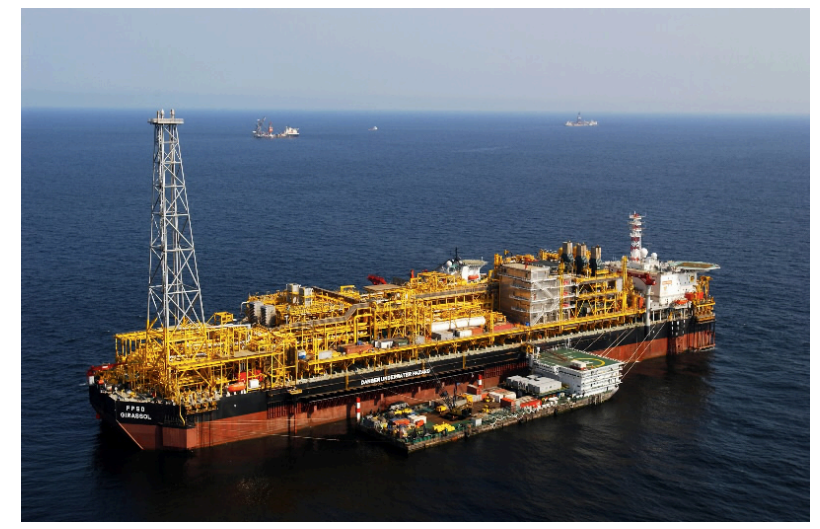

Figure 1: Photo of the FPSO on site.

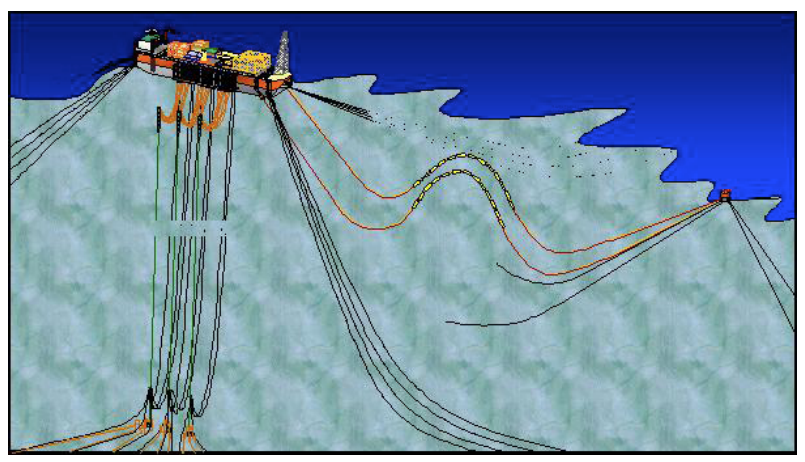

Figure 2: FPSO configuration including spread mooring, riser towers and export lines (Artist view).

\section{Environmental conditions: measurement and characterization}

The first step in the design process is to describe the local met-ocean conditions that will be used to generate the environmental loadings acting on the offshore structure. In the present case, project design values were available but they were not used directly. Instead, the design process was repeated from start to clearly identify the various uncertainties that arise at the different stages. The instrumentation to characterise the met-ocean conditions consisted of a Datawell Waverider Buoy, a Vaisala Maws anemometer and met-station, and two Acoustic Doppler Current Profilers (ADCPs) by RD Instruments (RDI) attached to a mooring line. The instrumentation was maintained on site from May 2002 until July 2004, thus offering 2 years of simultaneous wind / wave / current data. The wave buoy was kept in place for an additional year. The analysis of recorded met-ocean data showed that even if the maritime climate is mild in West Africa, the wave, wind and current conditions are complex and need to be analyzed carefully as described hereafter.

\subsection{Current analysis}

Current is at the origin of drag forces on both the FPSO hull and the mooring lines. A sub-surface current profile reduction was carried out to obtain time series of parameters characterising the current profile. Following a quality control and consolidation of the current database, a visual inspection of the current profiles showed the complexity and variety of the sub-surface currents. The data often exhibits relatively strong currents (up to $0.8 \mathrm{~m} / \mathrm{s}$ ) with sheared or rotating speeds as illustrated in Fig. 3. 

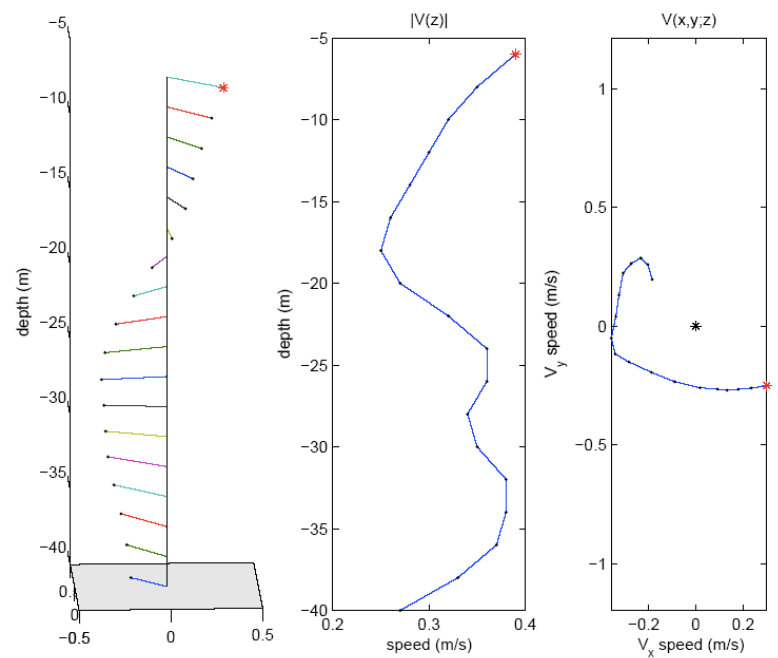

Figure 3: Example of measured sub-surface current profile.
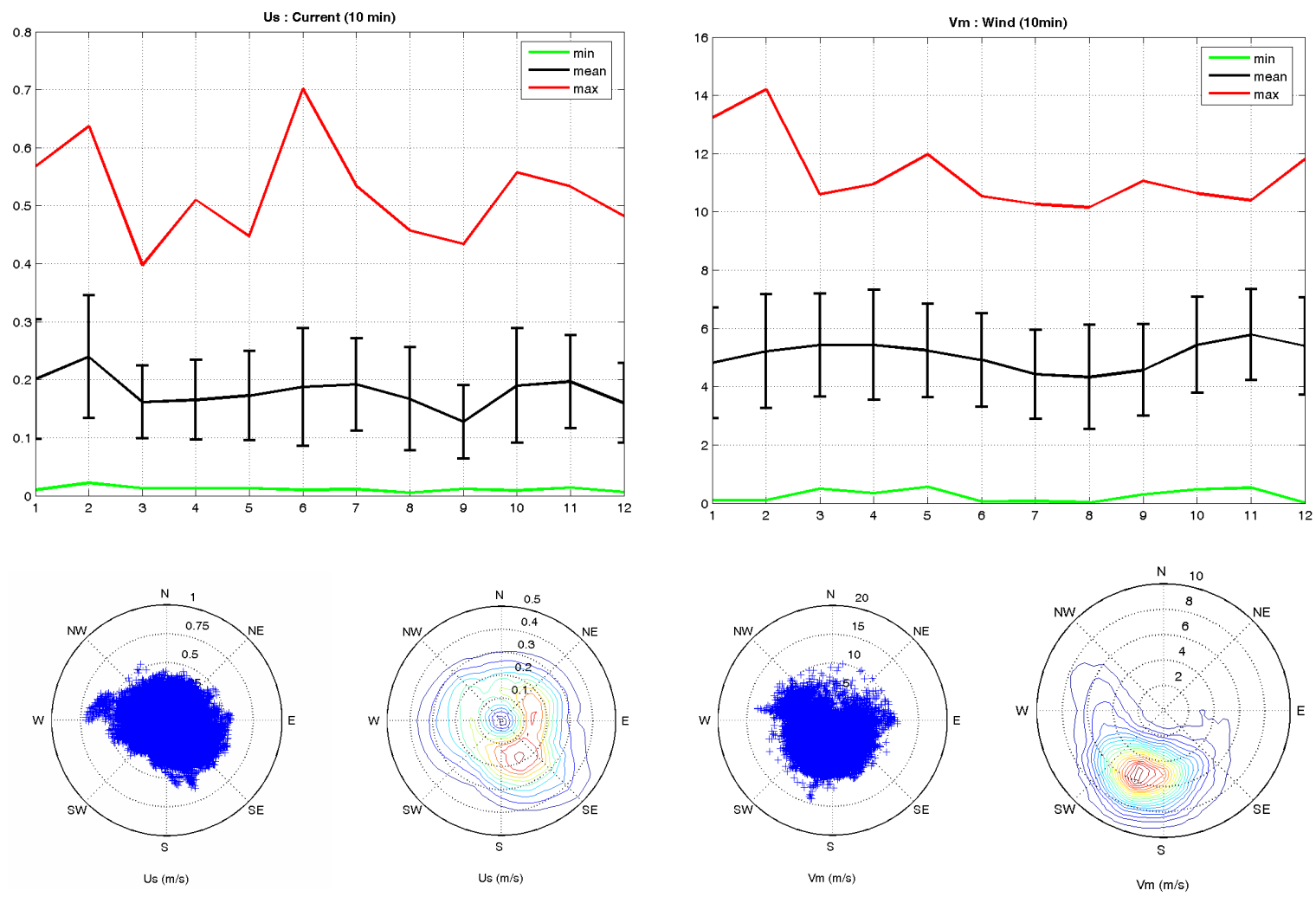

Figure 4: Monthly statistics, scatter plot and directional distribution of sub-surface current (Left) and wind (Right). 
Then, various methods were applied to characterise subsurface profiles with a limited number of parameters:

- A uniform profile (with the same direction over the FPSO draft);

- A sheared profile (with the same direction over the FPSO draft);

- An Empirical Orthogonal Function (EOF) decomposition also know as Principal Component Analysis (PCA) which had been previously applied with some success to described current profiles [10]. The method basically consists in a linear transformation of the data to a new coordinate system such that the greatest variance by any projection of the data comes to lie on the first coordinate, also called the first EOF mode, the second variance on the second EOF mode, etc.

Although the use of EOF decomposition with 3 modes would have minimized the error, the uniform profile was retained for the rest of the study for practical reasons associated to the use of standard hydrodynamic and structural software. Unfortunately, this assumption adds some uncertainty to the analysis. Fig. 4 (left) shows the monthly statistics (min, mean, max, stdev), the scatter plot and joint distribution $\left(U-\theta_{U}\right)$ of surface current velocity and direction.

\subsection{Wind analysis}

The wind is at the origin of a drag force on the hull of the FPSO above the seawater level and on the topsides. While processing wind records, it appeared that the statistical properties of the wind during squall events significantly differ from that for trade winds. Squall events were therefore removed from the database for the purpose of this study because they have to be considered as extreme events on their own. It should be kept in mind that accounting for squall events is of the outmost importance to assess the extreme response of the mooring system, but taking them into account required a specific study that is not reported here. The response of the FPSO to squall events will be reported in a separate article due to length constrains.

A modified Kaimal wind spectrum was adjusted to the trade wind data and was later on used to model the wind in the simulations. Monthly statistics (min, mean, max and stdev), scatter plot and joint distribution $\left(V-\theta_{V}\right)$ of wind velocity and direction are illustrated in Fig. 4 (right).

\subsection{Wave analysis}

\subsubsection{Wave system extraction}

Although the wave climate is mild offshore West Africa, it is a key aspect for designing floating structures because of the presence of crossed seas that may have a significant effect on the floater behaviour. Fig. 5 and 6 show an example of multiple swells and a local wind sea acting simultaneously with various periods and directions, thus the importance of accurately partitioning the sea-state into swells and wind sea systems.

The frequency-direction wave spectra were calculated using an Iterated Maximum Likelihood Method with MATLAB tools, from successive half-hour raw data and then averaged over one hour. Several spectral parameters were calculated from the raw data or from the wave spectra including spectral density, main direction and directional spreading as a function of wave frequency. In addition, the maximum wave height was also calculated. As shown in Fig. 5, multi-modal wave spectra were observed most of the time, exhibiting simultaneous swells and often a local wind-sea.

Sea state systems were initially derived from a partitioning of sea-state spectra based on APL Waves Analysis software. An example of partitioning is presented in Fig. 6 (Left). Measured wave systems were characterized with a maximum of 3 components. The Main Swell was associated with the highest significant height $H_{s}$ among the components; the Secondary Swell was characterized from the second or the third system with $T_{p}>9 s$ and the Wind Sea for $T_{p}<9 s$. A Quality Control was carried out and some adjustments were made in particular to correctly identify the secondary swell system.

Statistics on the occurence of sea state combinations presented in Table 1 show that $84 \%$ of the sea-states are composed of a main swell alone, or a main swell with a wind sea component. Wave partitioning resulted in the creation of time series covering the three and a half years of wave records for each of the reduced parameters including significant height $\left(H_{s}\right)_{i}$, peak period $\left(T_{p}\right)_{i}$, peak direction $\theta_{i}$ for each of the three wave systems identified (main and secondary swells, wind sea). 

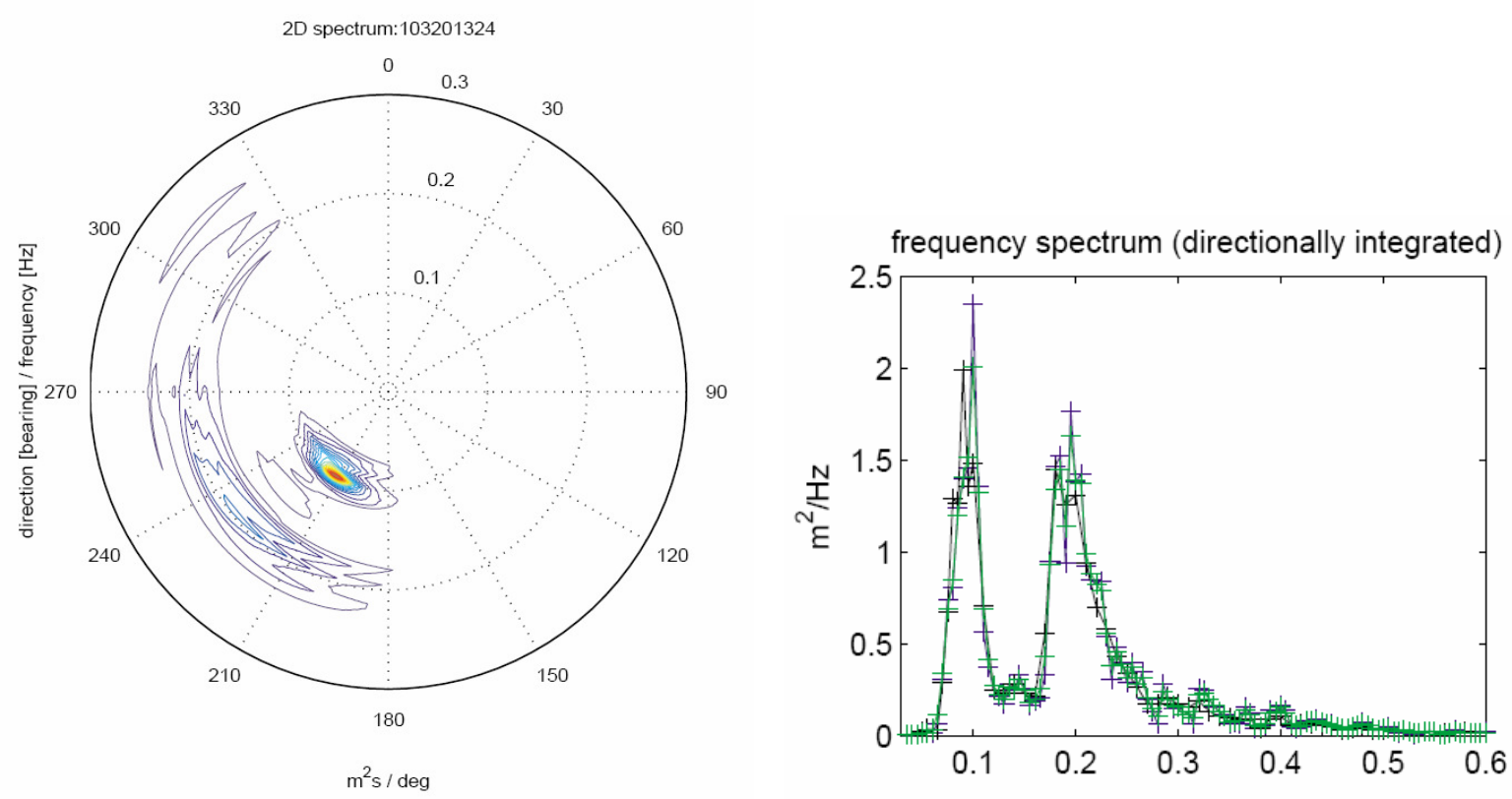

Figure 5: A typical multi-modal wave spectrum measured offshore Angola.
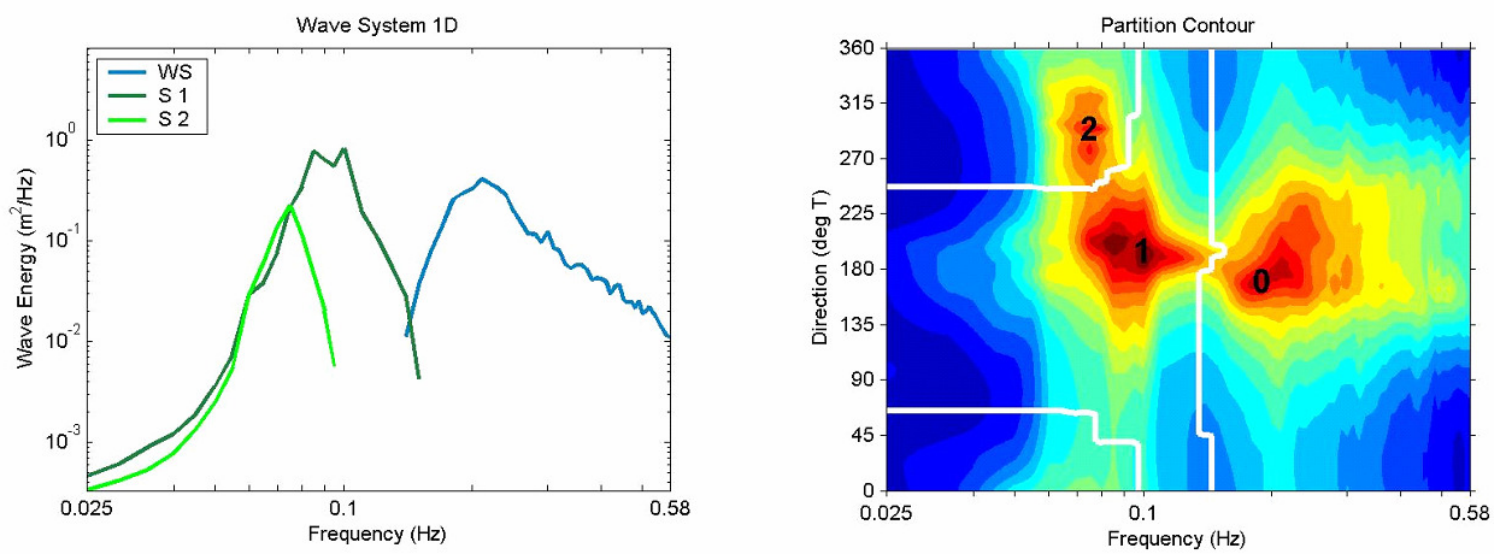

Figure 6: Example APL WAVES spectral partition plot from 17 Nov. 2001.

Table 1: Statistics of APL Wave Systems

\begin{tabular}{lc}
\hline Main Swell alone & $46.1 \%$ \\
Main Swell \& Second Swell & $8.2 \%$ \\
Main Swell \& Second Swell \& Wind Sea & $7.7 \%$ \\
Main Swell \& Wind Sea & $38.0 \%$ \\
\hline
\end{tabular}



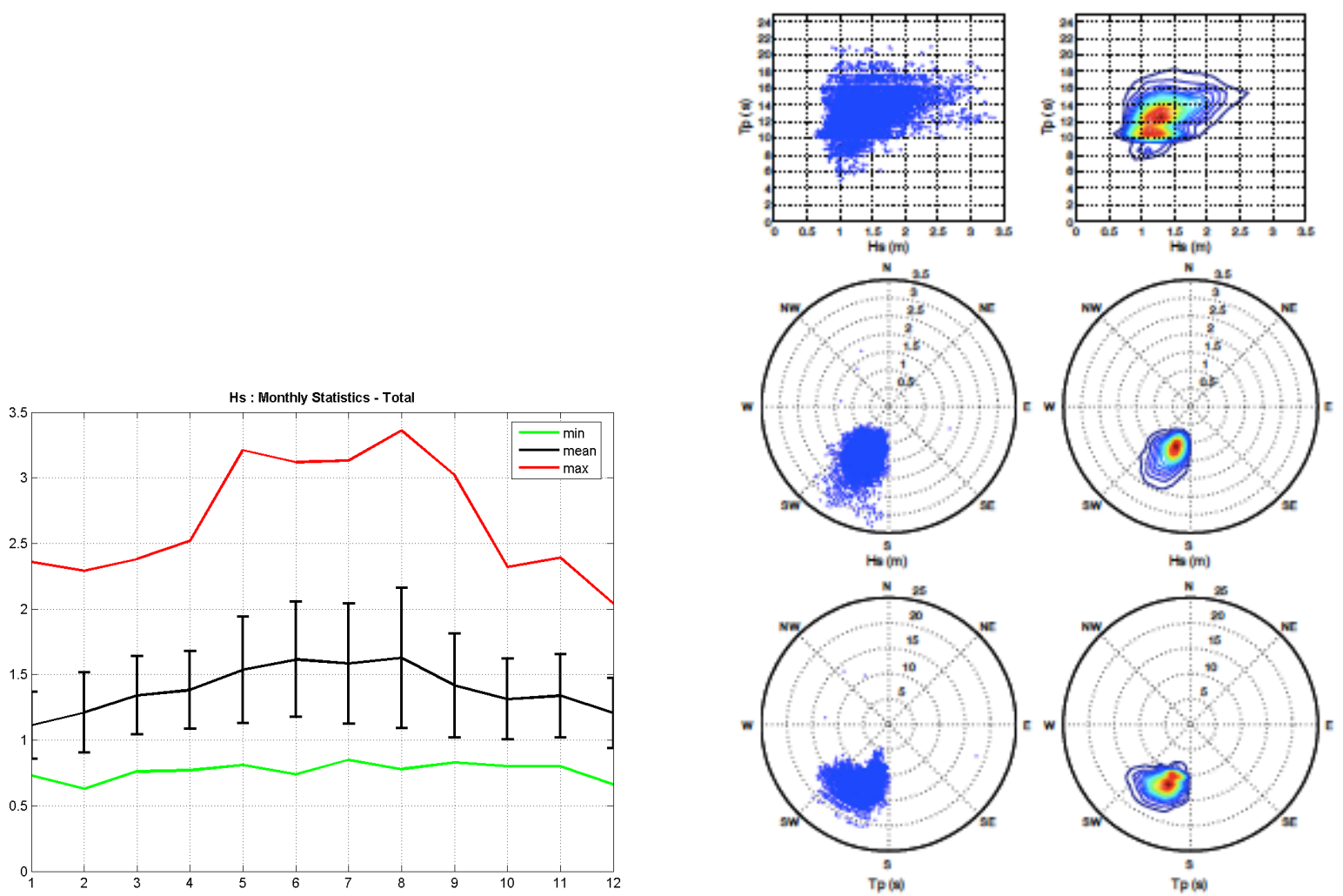

Figure 7: Statistics of total sea-state parameters.

\subsubsection{Wave system properties}

The parameters describing each wave system individually and the total sea-state were then analyzed to produce tables of statistics (min, mean, max, STD and quartiles), empirical distributions $H_{s}, T_{p}$ and the joint distributions $\left(H_{s}-T_{p}\right),\left(H_{s}-\theta\right),\left(T_{p}-\theta\right)$ and $\left(H_{s}-T_{p}\right)$ per directional sectors. Results of the statistical analysis are illustrated in Fig. 7 and 8. This last figure highlights the difference between main swell and wind sea significant wave heights, therefore justifying the choice to consider three separate wave systems to model the environment in a deterministic manner.

\section{Response Based Design}

\subsection{Methodology}

Response Based Design (RBD) methodology consists in running a large number of simulations to derive statistical estimates for the extreme values of the computed response. It requires the ability to generate a large enough database that describes realistically in a deterministic manner the marine environment. The use of the RBD methodology to design offshore structures is not new. Following the early work by Birades [11], the approach has had some success $[12,13,14,15,16]$ and is now partly included in design guidance [17]. Response Based Design often relies on the use of a Response Surface Model (RSM) to describe the behaviour of the structure [18, 19, 20, 21] and [22, 23, 24] for offshore structures. Response Based Design methodology was also applied to assess the extreme response of FPSOs [25, 26, 27, 28, 29, 30, 31, 32, 33, 34] though the approach remains strongly limited by the computational time required to perform a single simulation of the moored structure subject to environmental loadings. The required computational effort can be reduced by several orders of magnitude by decreasing the level of complexity of the 

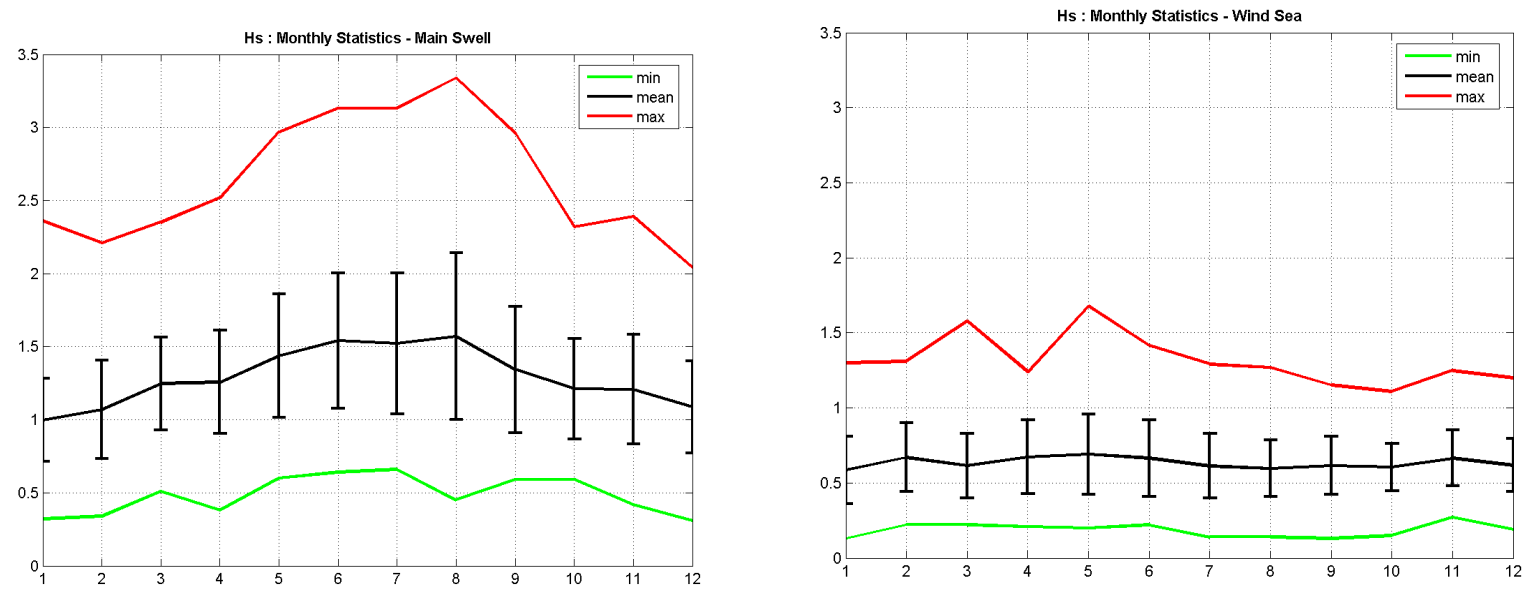

Figure 8: Monthly statistics of significant heights of main swells (Left) and wind seas (Right).

numerical model. Time domain fully coupled simulations are very demanding as the dynamics of the mooring lines has be to computed simultaneously with the motion of the floater. The required CPU time was in a ratio of circa one to one with the physical time which makes fully coupled simulations inappropriate for RBD unless a super-computer is used. The fully coupled dynamic analysis can be advantageously replaced by a quasi-static approach that neglects dynamic effects on the mooring lines and the risers, also assuming the later do not influence the FPSO motion. The classical analytical solution for catenary is then used to model the mooring lines that in turn influence the FPSO motion through their associated stiffness.

Such a simplification was performed as this assumption was justified in the present study. The met-ocean database consisted of approximately 25,000 data points for which the environmental conditions were recorded, covering a 3 years period of time. Direct simulations of the mechanical behavior of the spread moored FPSO with its mooring and riser systems were performed for all data points. Results of the direct simulations were then post-treated statistically to extrapolate an extreme value of the response for a given value of the return period.

\subsection{Description of the numerical model}

A direct simulation presently consists in a low-frequency time domain simulation with superposition of the wave frequency motion obtained from the motion RAOs (Response Amplitude Operator), assuming a quasi-static behavior of the mooring lines. The numerical model used to perform the direct simulation is therefore similar to the one used in the standard design methodology. The FPSO is modeled as a solid with 6 degrees of freedom to which are applied the following loadings:

- Inertia loads are applied at the centre of gravity (COG) and computed from the design data for the lightweight FPSO and the monitored level in each tank.

- Hydrodynamic loads are computed from the geometry using a diffraction radiation software [35]. Loads are separated into (i) radiation loads (added mass, radiation damping), (ii) excitation loads (diffraction + Froude Krylov loads) and (iii) low frequency loads calculated from the drift forces using Newman approximation.

- Wind and current loads are modeled by a Morison formulation using aerodynamic and hydodynamic drag coefficients issued from wind model tests.

- Anchoring loads are modeled by a set of non-linear springs imposing the quasi-static restoring force to the FPSO according to its actual position.

- Riser and export line loads are modeled as constant loads due to the catenary configuration of the jumpers. 


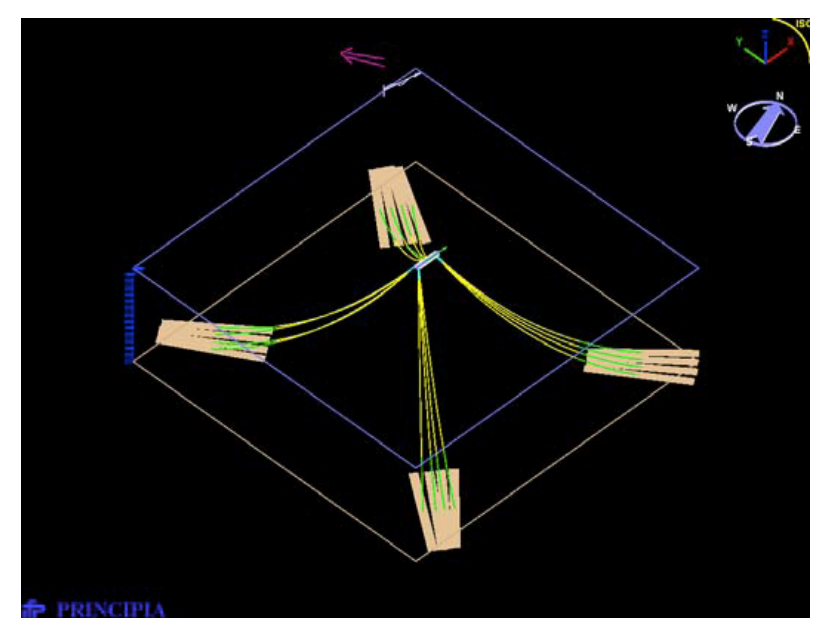

Figure 9: DeepLines ${ }^{T M}$ FEM model of the FPSO and its riser and mooring systems

Time domain simulations were performed using the model shown in Fig. 9. Wave frequency motion and low frequency motion are superimposed and a quasi-static approach is used for estimating dynamic tensions, which may not always be appropriate as the dynamics of the mooring system may influence the FPSO position when severe environments are addressed [36, 37]. Additional monitored data are required to build up an accurate model. In particular, effective values for cargo tank and ballast tank filling ratio, draft and attitude of the ship have been considered. All the previously mentioned data were simultaneously available over a one-year period of time. Values issued from the design stage are mainly wind and current polar coefficients obtained from wind tunnel model tests. Hydrodynamic data are computed using 3D diffraction radiation model [35] for a matrix of 5 drafts by 5 trim angles, which is then used for interpolation.

\subsection{Validation of the model}

Classicaly, time domain simulations are run over a 3-hour period of time during which the statistical properties of the sea state are considered to be constant. As the FPSO motion natural period for horizontal motions (surge and sway) is of the order of 300s, transient effects are expected to arise during the first hour of the simulation. Only results from the last hour of the simulation were therefore retained. The model was calibrated and benchmarked against measurements. Fig. 10 shows a comparison for the maximum tension in a mooring line as predicted by the numerical model and as measured. The reasonable agreement indicates that the numerical model is correctly calibrated and the input data are consistent. Tentative validation of the FPSO motion was made but the Dynamic Global Positioning System (DGPS) did not provide enough accuracy to have tangible results.

As the simulations were performed in the time domain using a random wave sequence with prescribed energy spectrum, the sample variability makes it difficult to interpret directly the maximum value that is being computed. Part of the discrepancies observed in Fig. 10 can be explained by the definition that has been used for the maximum value. To obtain a representative value of the maximum tension, the recommended practice [3] is to repeat the simulation using different seeds to initialise the wave sequence. Alternatively statistics can be performed on sub-windows of the simulated time period. In the present study, the last hour of each simulation was analyzed as follows: six windows of ten minutes were defined and the maxima over each window were recorded. By analogy with classification rules, the so-called design value was set equal to the maximum value over all windows plus two times the standard deviation of the observed block maxima:

$$
\Delta X_{\text {design }}=\Delta X_{\max }+2 \sigma_{X_{\max }}
$$

where $\Delta X_{\max }$ is the maximum value that has been computed and $\sigma_{X_{\max }}$ is the observed standard deviation of the observed maxima over the six windows.

\subsection{Extreme analysis of simulation results}

The reponse of the FPSO subject to the measured enviromental loads was computed and recorded during one year. Simulations were performed every 10 minutes to match the measured sampling rate for wind loads, leading to 


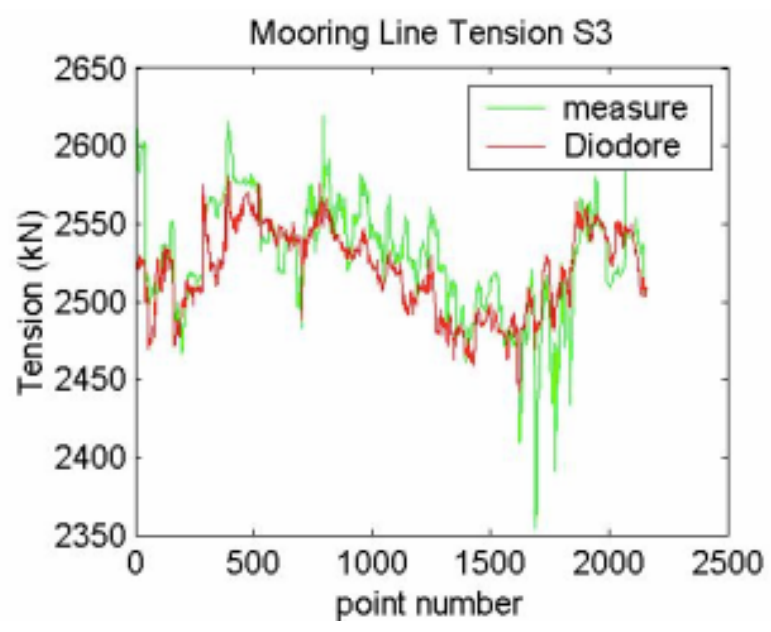

Figure 10: Comparison between measured and predicted value of the mooring line tension.

\begin{tabular}{lcc} 
Table 2: Main statistics for FPSO motion during the year of simulation \\
\hline 10 minutes sub-windows & surge $(\mathrm{m})$ & sway $(\mathrm{m})$ \\
Minimum & -12.858 & -8.207 \\
1st Quantile & -2.319 & 4.468 \\
Median & -1.782 & 5.459 \\
Mean & -2.167 & 5.16 \\
3rd Quantile & -1.450 & 6.37 \\
Maximum & -0.215 & 8.146 \\
\hline
\end{tabular}

approximately 25,000 calculations in total corresponding to several days of CPU time on a standard PC. Only the results of interest (FPSO offset and maximum tension in each mooring line) were stored and post-process afterward.

The derivation of extreme values is a key point in both classical or Response Based Design methodology as it is at the origin or at the conclusion of the method. All the distributions were computed as univariate distribution and were then fitted by probabilistic distribution models. No assumption on the type of extreme law that should be fitted on the results was made among Generalized Extreme Value (GEV) distributions, which includes Weibull maximum and Gumbel distributions. The fitting used the maximum likelihood estimates. Derivation of the extreme values for sea state parameters and therefore for all the associated responses such as offset and tension requires independence of the observed maxima used in the empirical distribution. The weekly maxima were used after a sensitivity analysis was performed. The response extrapolation process is illustrated in Fig. 11 and results are presented in Table 2. The later will be discussed at the end in Section 7

\section{Statistical description of the environment}

So far, the main parameters characterising the marine environment at the site location have been extracted from field measurements. A database compiling the simultaneous values of these parameters over a two-year period of time was built (Section 2) and used as input to perform direct simulations (Section 3). In order to perform the design of the mooring system within the framework of reliability analysis, it is however necessary to create a statistical representation of the marine environment instead of a deterministic one.

This section presents the statistical analysis of the met-ocean data. The dependence between the various met-ocean variables is studied first, prior to deriving the marginal distribution and joint distribution when needed. The problem of deriving joint distributions of met-ocean conditions has led to numerous studies since the early work of Winterstein $[38,39,40,41]$, see e.g. [42, 43, 44, 45, 46, 47, 48, 49, 50, 51, 52, 53]. The originality here was to include in the 


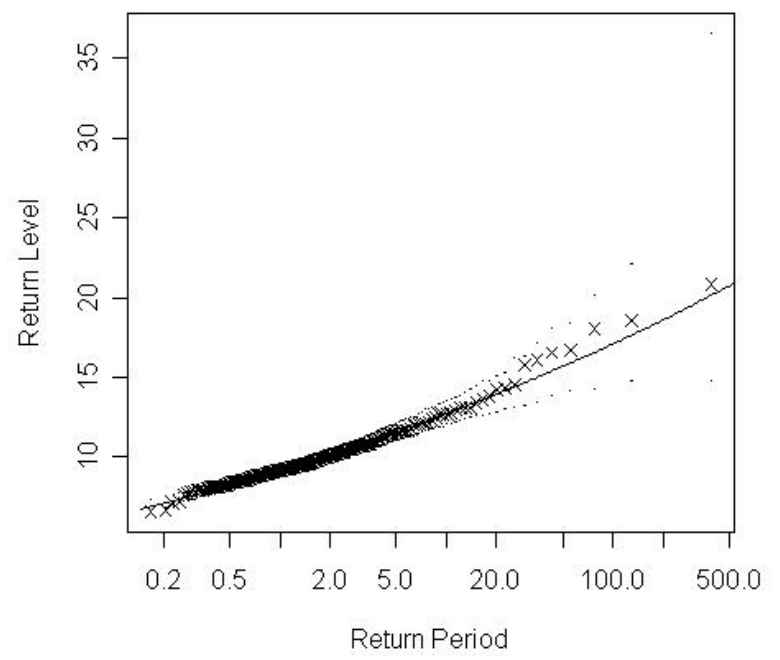

Figure 11: Maximum offset extrapolation from RBD. The Return Level corresponds to the FPSO Offset (m).

approach the dependence with respect to direction (Section 4.2) and to propose a method to model accurately both the body and the tail of the marginal distribution (Section 4.3).

Once the various distributions have been modeled, the Inverse First Order Reliability Method (I-FORM) allows calculating extreme environmental contours for a given return period from the joint distributions of several variables. Iso-contours associated to a probability of non-exceedence $p$ related to the specified return period are then searched for the points that maximize some response function. An introduction to the method is given in Winterstein et al. $[38,39]$ and further studies have been performed since then, see e.g [54, 55, 56, 57, 58]. Section 4.3.1 describes a practical methodology to enforce that the extreme contours are consistent with the extremes calculated for each variable.

\subsection{Dependence analysis and joint probabilities}

\subsubsection{Dependence between the respective intensities}

The first step of the analysis was to describe the marginal PDF of the main variables i.e. the variables characterising the intensity of each met-ocean component including current $(U)$, wind speed $(V)$, and significant height $H_{s}$ of each individual wave system. Their empirical distributions were fitted by probability distribution models, Gumbel and Weibull, using a least square method that minimizes the error between the empirical cumulative distribution and the fitted model. The analysis highlighted the need for two types of marginal distribution models, one giving the best fit of the bulk of the distribution and another for adjusting the highest values as described in Section 4.3.

The second step was to analyze the dependence between variables, using three methods:

- Statistical test of significance of correlation: Bravais-Pearson and Spearman.

- Scatter plot between two variables $\left(X_{1}\right.$ and $\left.X_{2}\right)$ and analysis of main conditional statistical parameters (mean and standard deviation) of one variable versus the other.

- Principal Component Analysis (PCA) that allows for the analysis of dependence between more than 2 variables.

The dependence analysis resulted in following conclusions:

- Independence of sub-surface currents versus wind and sea states;

- Independence between sea state systems;

- Independence of wind and swell systems; 

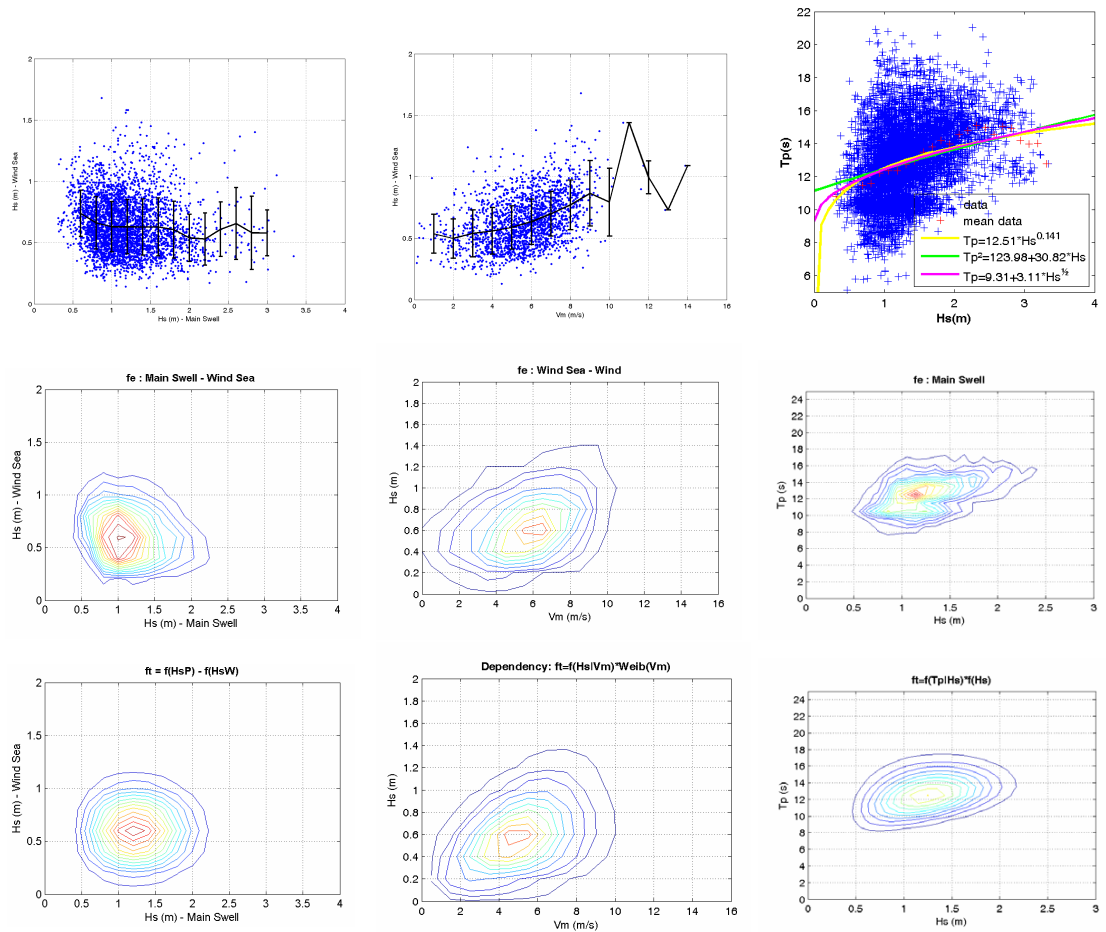

Figure 12: Scatter-plot, empirical joint distribution and model joint distribution for independent variables (Left) and two sets of correlated variables (Center and Right), namely (Main Swell,Wind Sea), (Wind Sea, Wind velocity) and $\left(H_{s}, T_{p}\right)$.

- Dependence of wind and wind sea (intensity and direction);

- Dependence of $H_{s}$ and $T_{p}$ in every sea state system.

Examples of un-correlated and correlated variables are illustrated in Fig. 12. When dependence was observed, conditional distributions were built using standard models such as Weibull or Lognormal.

\subsection{Dependence with respect to direction}

Dependence of the intensity of the loading with respect to direction was investigated. The symbols in Fig. 13 represent the observed directional distribution for current and wind speed for a 22.5 degrees direction interval. The observed directional distribution for main swell is also plotted in Fig. 14 for a 7.5 degrees direction interval. These figures clearly show the strong directionality of the environment in this region of the world and stress the need to use analytical models that have the ability to represent accurately the directionality of the environment. From a mathematical point of view, the directional PDF were represented by a combination of a uniform PDF and a number of Gauss PDF as described in Section 4.4.1. Such a description was not reported before to the knowledge of the authors.

This fitting process is explained hereafter for the case of the surface current $U$. In order to match the three distinct peaks of the observed directional distribution shown in Fig. 13, the analytical distribution was sought in the form of the combination of a uniform PDF and 3 normal Gauss PDF which parameters were identified by least squares method. The fitted distribution is represented by the red line in Fig. 13. The current velocity distribution was then defined as conditional to the current direction. A Gumbel model was adjusted to each current speed PDF per directional sector. The empirical directional Gumbel parameters were numerically adjusted using the same analytical representation as for the current direction PDF, i.e., a combination of a constant uniform PDF and 3 normal Gauss PDF. This model enables to achieve a complete Inverse-FORM computation, although it works mainly because the design points are close to the dominant directions of the met-ocean variables, which means that the possible issue of extrapolating to extremes the directional distributions was not raised. 

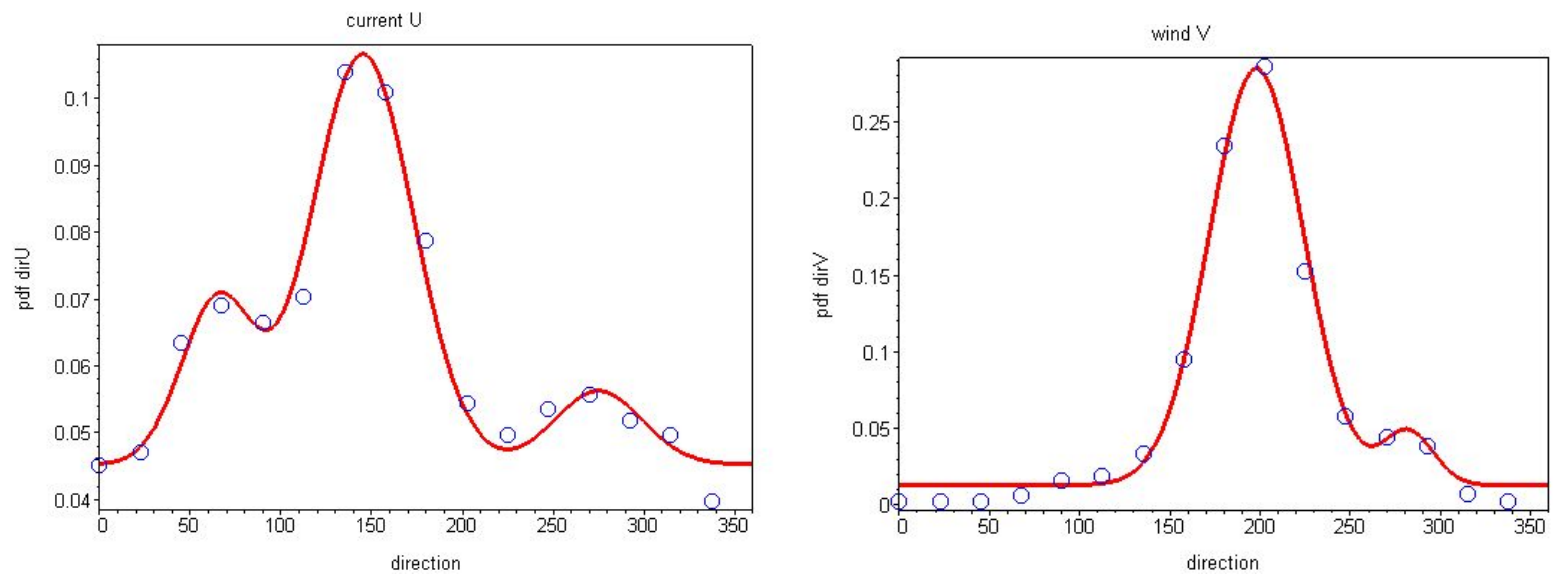

Figure 13: Directional distribution of current (left) and wind (right) direction.

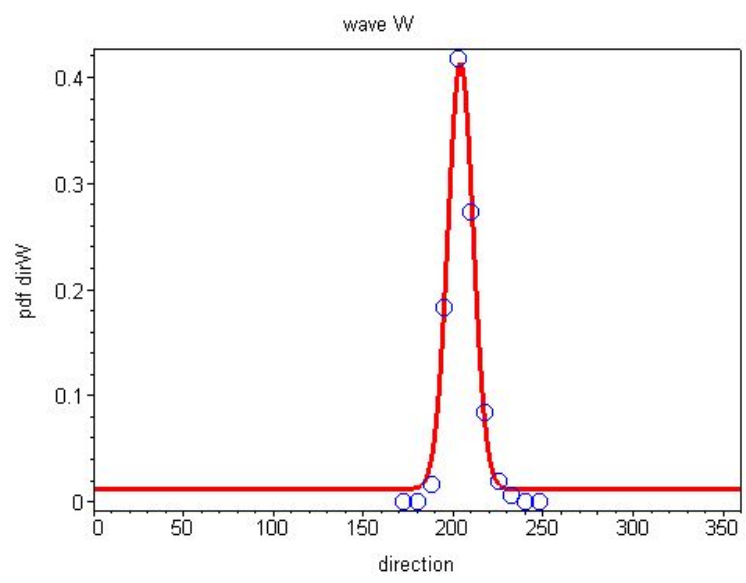

Figure 14: Directional distribution of wave direction

A fairly similar method was applied to wind direction and wind speed conditional to direction. On the other hand, due to the narrowness of the main swell direction distribution, the swell wave height was assumed to be independent from swell direction. The swell directional PDF was set to zero out of a narrow angular sector around the dominant direction.

\subsection{Joint Extremes}

\subsubsection{Corrective extreme factors}

In the Inverse-FORM methodology, the distribution model shall be representative of the global distribution of each variable, including the distribution of the highest values laying in the tail of the distribution. The extreme contours shall be able to represent extreme values of a given variable associated to normal conditions of another variable.

Furthermore, the extreme contours shall be consistent with the extremes calculated for each variable, which may lead to some difficulties in the application of a standard Inverse-FORM methodology, because the extremes are often calculated on the basis of distributions of peak values (Peak over Threshold method), or distributions of maximal values over given periods of time (e.g. extrapolation of annual maxima). The following practical method was applied to overcome these difficulties:

- Step 1: Two types of marginal distribution models of main variables $U, V$ and $\left(H_{s}\right)_{i}$ were considered to lead to a good representation of the global distribution but also of the severe conditions, because this is required for 

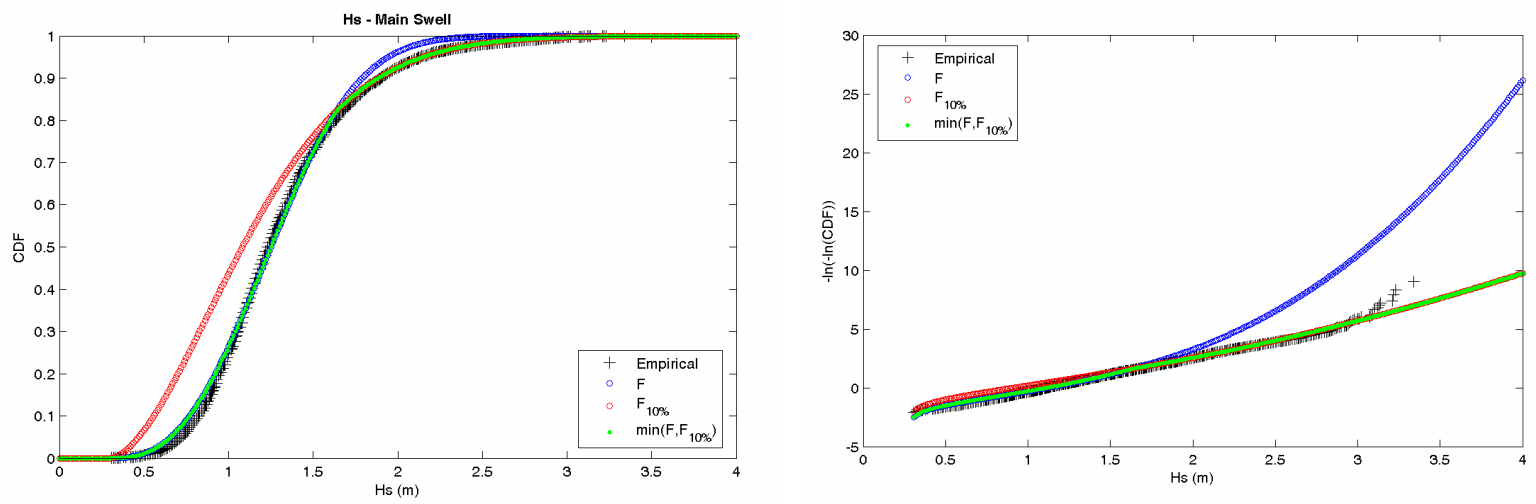

Figure 15: New distribution model of $H_{s}$ (Main Swell).

the derivation of extreme contours: $F(X)$ the best fits of the bulk of the data cumulative distribution function $(\mathrm{CDF}), F_{10 \%}(X)$ the fits of the $10 \%$ highest values, i.e. the tail of the distribution, for modelling of severe environmental conditions. It should be noted that the selection of a $10 \%$ threshold is somewhat arbitrary. For each main variable, a new distribution model was set-up (Fig. 15) introducing $x_{t}$ defined as:

$$
F\left(X<x_{t}\right)=F_{10 \%}\left(X<x_{t}\right)
$$

and defining a matched distribution such that:

$$
\begin{array}{r}
P(X<x)=F(X<x) \quad \text { for } \quad X<x_{t} \\
P(X<x)=F_{10 \%}(X<x) \quad \text { for } \quad X \geqslant x_{t}
\end{array}
$$

- Step 2: A corrective extreme factor was associated to each main variable, so that the extremes calculated from the distribution coincide with the considered return value calculated for each variable separately: If $P(X)$ is the revised distribution of the variable $X$, if the 100-y extreme (for instance) is $x_{100}$, and if the number of events is $N$ (e.g. $100 * 365.25 * 8$ for $H_{s}$ at a time step of 3 hours), then a corrective factor $k$ is applied so that:

$$
P\left(X>x_{100}\right)=1-\frac{1}{k N}
$$

leading to a correction of the cumulative distribution $P(X)$ :

$$
P_{c}(X>x)=1-k(1-P(X>x)), \quad \text { or } \quad P_{c}(X<x)=k P(X<x)
$$

As defined, $P_{c}$ is no more a cumulative distribution, but over the levels we are interested in, $k$ could be supposed to be $x$ dependent and to tend to 1 . When the time steps of two variables are not the same (e.g. $H_{s}$ at time steps of 3 hours and $V$ at time steps of 10 minutes), the CDF must be adapted in order to get extreme contours that are consistent with extremes calculated on different time bases. If $X_{1}$ is the variable defined on the longer basis, say a duration $D_{1}$, a new set of variables is derived from the second variable: $X_{2 m}=\max \left(X_{2}\right)_{D_{1}}$ then, a new distribution model of $X_{2 m}$ is built, and the same approach as above is applied.

- Step 3: Then, the standard I-FORM methodology can be applied on the basis of the $2 \mathrm{CDF}, P_{c 1}\left(X_{1}\right)$ and $P_{c 2}\left(X_{2}\right)$. With the methodology presented above, the two CDF were built to get conservative contours consistent with extreme values (at least the 100-y contour with the 100-y extremes). 

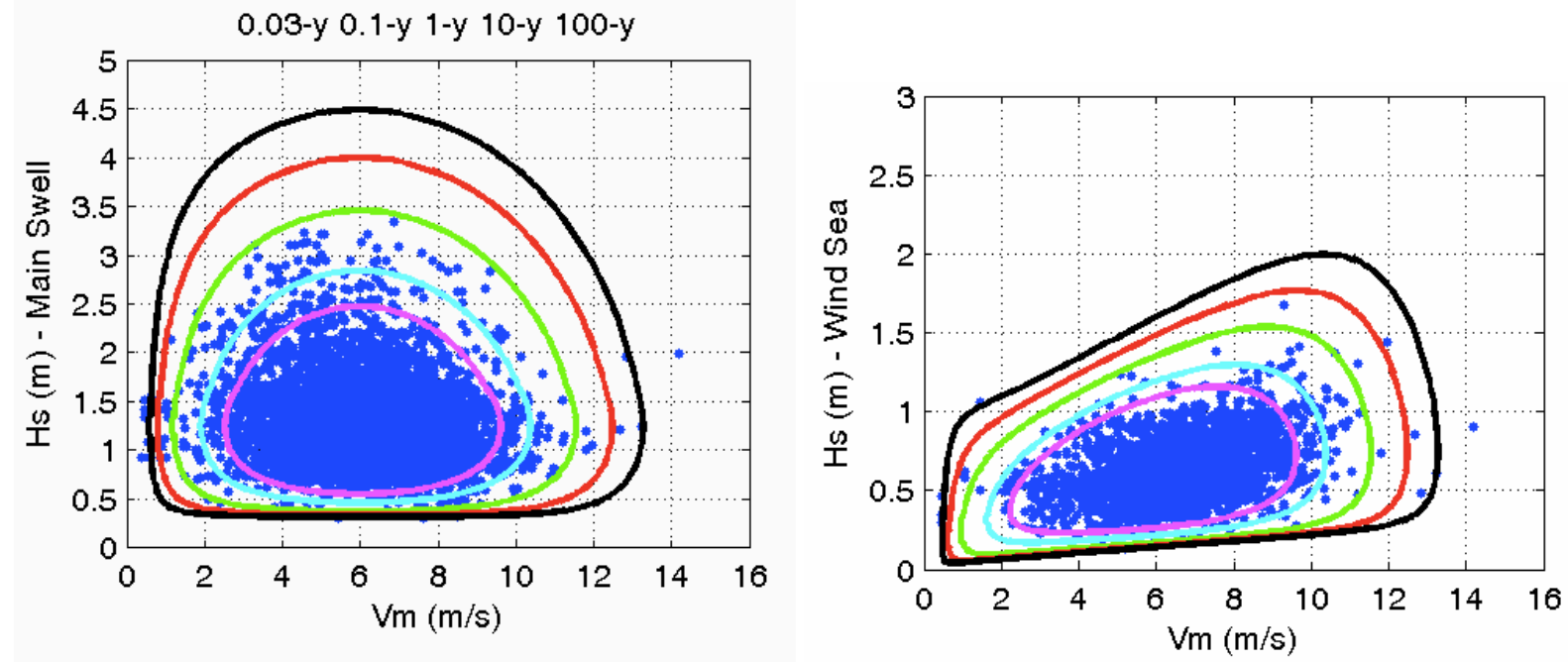

Figure 16: Examples of Inverse-FORM contours for Hs main swell - Wind (left) and Hs Wind Sea and Wind (rigth).

\subsubsection{Inverse-FORM contours}

Exemples of use of the Inverse-FORM contours are provided in [54, 55, 56, 58, 57]. In the space of the standard normal variables, the extreme environmental contour with a $p$-probability is the hypersphere (a circle in 2D) with a radius $\beta=\Phi^{-1}(1-p)$, where $\Phi$ is the Gauss cumulative distribution. The standard normal variables are calculated by the Rosenblatt transformation [59]: if $X$ is a physical random vector (e.g. $\left(H_{s}, T_{p}\right)$ ), a standard normal vector $U=T(X)$ is obtained, where $T$ is the Rosenblatt transformation. The variables on the circle of p-probability in the standard normal space are $U_{c}$. To get the values of these variables in the physical space (I-FORM contours), the inverse Rosenblatt transformation is applied:

$$
X_{c}=T^{-1}\left(U_{c}\right)
$$

Based on the analyses described in the previous sections, the Inverse-FORM methodology was applied. This led to joint models for several couples of variables. Some Inverse-FORM contours resulting from this analysis are presented in Fig. 16.

\subsubsection{Limitations}

This study has led to joint extreme criteria that can be used for design. However, some limitations in the method and some uncertainties must be stressed. In particular, squall winds were excluded from the study and currents representation was simplified. But the main difficulties came from the application of I-FORM methodology, in particular on following topics:

- The necessity to use two distribution models to fit the empirical data, one for the bulk of the distribution and another for adjusting the highest values;

- The necessity to use corrective extreme factors in the distribution models, in order to remain consistent with extreme values;

- The extrapolation to 100 years from only 3 years of data and the choice of the law $F_{10 \%}$;

- The potential influence of seasonal dependence between parameters;

- The method to get directional contours, which is still being debated;

- The need to simplify the contours for design purposes. 
- The first order approximation in FORM method.

In addition, we must emphasize the fact that the I-FORM methodology was applied to pairs of variables only. It must be stressed that it is more difficult to deal with multivariate statistics in higher dimensions than the two considered in this study, particularly when the variables are dependent.

\subsection{Statistical model of the environment}

\subsubsection{Choice of the key variables}

The environment is composed of the current speed and direction, the wind speed and direction and the three wave systems with their associated wave height, peak period and directions. Each sea state is therefore described by a total of thirteen random variables. In order to reduce the number of variables but still retaining the main ones, it is interesting to study the response of the system to the different environmental loadings. Such a study is presented in Section 5. The number of relevant state variables to be included into the First Order Reliability Method is governed by the adequacy of the Response Surface Models to represent correctly the behavior of the mechanical system and its sensitivity to the environmental variables. When deriving the Response Surface Model, it was observed that only current, wind and main swell had a significant effect on the FPSO offset and mooring line tension. Consequently, only 7 physical variables as shown in Table 3 were retained in the statistical model for the environment:

- The surface current was considered as a physical phenomenon modeled by a random process that could be described by means of 2 random variables, i.e. the direction and the velocity.

- The wind was here considered as a physical phenomenon modeled by a random process, which could be described by means of 2 random variables, i.e. the direction and the velocity.

- The main swell was considered as a physical phenomenon modeled by a 3-hour sea states described by means of 3 random variables: the direction, the significant height and the peak period.

\begin{tabular}{lcc}
\multicolumn{3}{c}{ Table 3: Random variable definition } \\
Designation & Symbol & Random variable \\
\hline Current direction & $\operatorname{dir} U$ & $x_{1}$ \\
Current velocity & $U$ & $x_{2}$ \\
Wind direction & $\operatorname{dir} V$ & $x_{3}$ \\
Wind velocity & $V$ & $x_{4}$ \\
Wave (main swell) direction & $\operatorname{dir} W$ & $x_{5}$ \\
Significant wave height & $H_{S}$ & $x_{6}$ \\
Peak period & $T_{p}$ & $x_{7}$ \\
\hline
\end{tabular}

\subsubsection{Current}

A simple Gaussian model was used to fit the current directional PDF as the combination of a constant function and 3 Gauss functions for $x_{1} \in[0,2 \pi[$ :

$$
P_{\text {dirU }}\left(x_{1}\right)=p_{10}+\sum_{1 \leq k \leq 3} \phi_{1 k}\left(x_{1}\right), \quad \text { where } \quad \phi_{1 k}\left(x_{1}\right)=\exp \left[-\frac{1}{2}\left(\frac{x_{1}-\theta_{1 k}}{\sigma_{1 k}}\right)^{2}\right]
$$

All parameters present in the current direction analytical PDF have been numerically determined by least squares methods applied to measured data. For the current velocity, Gumbel laws were the best fit for measured distributions. A 2-parameter Gumbel law was used to represent the cumulative distribution function of the current velocity with constant parameters in absence of directional effect or with variable parameters in case of directional effects.

- Model without directional effect: the current velocity distribution was considered as independent upon the current direction and the Gumbel parameters $a_{U_{0}}$ and $b_{U_{0}}$ were considered as constant:

$$
P_{U}\left(x_{2}\right)=\exp \left[-\exp \left(\frac{x_{2}-a_{U_{0}}}{b_{U_{0}}}\right)\right]
$$


- Model with directional effects: the current velocity distribution was conditional to the current direction with the Gumbel parameters $a_{U}$ and $b_{U}$ function of the current direction:

$$
P_{U}\left(x_{2} \mid x_{1}\right)=\exp \left[-\exp \left(\frac{x_{2}-a_{U}\left(x_{1}\right)}{b_{U}\left(x_{1}\right)}\right)\right]
$$

For every direction present in the measured data, the empirical directional parameters $\left(a_{U}\right)_{\exp }$ and $\left(b_{U}\right)_{\exp }$ were obtained from measured data using iterative maximum likelihood methods. The directional parameters $a_{U}$ and $b_{U}$ were numerically adjusted from the empirical directional parameters using the same analytical representation as for the current directional PDF, i.e., a combination of a constant function and 3 Gauss functions, with identical principal directions and standard deviations as for the measured directional PDF:

$$
a_{U}\left(x_{1}\right)=a_{10}+\sum_{1 \leq k \leq 3} a_{1 k} \cdot \phi_{1 k}\left(x_{1}\right), \quad \text { and } \quad b_{U}\left(x_{1}\right)=b_{10}+\sum_{1 \leq k \leq 3} b_{1 k} \cdot \phi_{1 k}\left(x_{1}\right)
$$

The parameters $a_{1 k}$ and $b_{1 k}$ were numerically determined by linear regression methods applied to empirical directional parameters.

\subsubsection{Wind}

A simple Gaussian model was also used to fit the directional PDF for the wind speed as the combination of a uniform function and 2 Gauss functions for $x_{3} \in[0,2 \pi[$ :

$$
P_{d i r U}\left(x_{3}\right)=p_{30}+\sum_{1 \leq k \leq 3} p_{3 k} . \phi_{3 k}\left(x_{3}\right) \quad \text { where } \quad \phi_{3 k}\left(x_{3}\right)=\exp \left[-\frac{1}{2}\left(\frac{x_{3}-\theta_{3 k}}{\sigma_{3 k}}\right)^{2}\right]
$$

All parameters present in the wind direction PDF were numerically determined by least squares methods from measured data. For the wind velocity, Weibull laws were the best fit for measured distributions. A 3-parameter Weibull law was used to represent the cumulative distribution function of the wind velocity with constant parameters in absence of directional effect or with variable parameters in case of directional effects.

- Model without directional effect: The wind velocity distribution was considered as independent upon the wind direction. The Weibull parameters $a_{V_{0}}$ and $b_{V_{0}}$ were set constant.

- Model with directional effects: The wind velocity distribution was considered as conditional to the wind direction. Only one Weibull parameter (the scale parameter) was function of the wind direction:

$$
P_{\operatorname{dir} V}\left(x_{4} \mid x_{3}\right)=1-\exp \left[-\left(\frac{x_{4}-a_{V_{0}}}{b_{V}\left(x_{3}\right)}\right)^{c_{V_{0}}}\right]
$$

For every direction present in the measured data, the empirical directional parameter $\left(b_{V}\right)_{\exp }$ was computed from measured data using iterative maximum likelihood methods. The scale parameter, $b_{V}$, has been numerically adjusted from the empirical directional parameter using the same analytical representation as for the current direction PDF, i.e., a combination of a constant function and 2 Gauss functions, with the same principal directions and standard deviations. The parameters $b_{3 k}$ were numerically determined by linear regression methods. The other two Weibull parameters were kept constant due to their small variations.

$$
b_{V}\left(x_{3}\right)=b_{30}+\sum_{1 \leq k \leq 3} b_{3 k} \cdot \phi\left(x_{3}\right)
$$

\subsubsection{Wave (Main Swell)}

As the measured directional distribution for the wave plotted Fig. 14 contains a single peak into one principal direction, a single Gauss PDF was used to represent the PDF:

$$
P_{d i r W}\left(x_{5}\right)=p_{5} \cdot \exp \left[-\frac{1}{2}\left(\frac{x_{5}-\theta_{5}}{\sigma_{5}}\right)^{2}\right]
$$


Due to the narrowness of the wave direction distribution, a Weibull law with 3 constant parameters was chosen to represent the significant height PDF [6]. The 3 parameters were taken as constant and independent from the wave direction:

$$
P_{H s}\left(x_{6}\right)=1-\exp \left[-\left(\frac{x_{6}-a_{H_{s}}}{b_{H_{s}}}\right)^{c_{H_{s}}}\right]
$$

The peak period distribution was modelled as a lognormal law with mean value and standard deviation dependant upon significant height:

$$
P_{T_{p}}\left(x_{7} \mid x_{6}\right)=\Phi\left(\frac{\ln \left(x_{7}\right)-a_{T_{p}}\left(x_{6}\right)}{b_{T_{p}}\left(x_{6}\right)}\right)
$$

with conditional parameters:

$$
a_{T_{p}}\left(x_{6}\right)=\ln \left(a_{T p_{0}}+a_{T p_{1}} \sqrt{x_{6}}\right) \quad \text { and } \quad b_{T_{p}}\left(x_{6}\right)=\sqrt{b_{T p_{0}}+b_{T p_{1}} \exp \left(-b_{T p_{2}} \cdot x_{6}\right)}
$$

\section{Response Surface Model}

Response Surface Models for the FPSO offset and the tension in the mooring lines were build in order to decrease the CPU time required by First Order Reliability Method to converge to the design point. RSM have been applied with some success to describe the behaviour of offshore structures [23, 24] as well as that of other types of structures $[18,21]$. The RSM allows characterising the response of the FPSO by mean of simple (polynonial) formulae involving the main parameters [27, 28]. The present study made use of RSM in conjonction with First Order Reliability Method, as described in Section 6.

The usual procedure for deriving a RSM is to choose an analytical representation of the solution and to identify the coefficients by mean of a fitting method such as least squares method. The most frequently used functions are quadratic forms, i.e. second-order polynomials of the basic variables, as such an analytical representation locally matches with the Taylor expansion of the solution. In the present case, RSM were sought in term of variations around an equilibrium configuration and a distinction was made between the steady $\left(X_{s}\right)$ and unsteady $\left(X_{u}\right)$ parts of the response according to:

$$
\Delta X(t)=\Delta X_{s}+\Delta X_{u}(t)
$$

Analysis of data indicated that the characteristic time scales for variations in current/wind speed and direction were longer than the first natural period of the system so that this assumption was fully justified for the current $\left(U, \theta_{U}\right)$ and may be justified for wind $\left(V, \theta_{V}\right)$ disregarding squall events.

Going further into the decomposition of the different components of the response, it is proposed to split contributions of current and wind in the one hand, and wave systems in the other hand. In other words, the steady part depends on steady loads $\left(U, \theta_{U}, V, \theta_{V}\right)$ and the unsteady part, due to waves, is a function of $\left(H_{s}, T_{p}, \theta_{W}\right)$ for the three wave systems that have been considered. Assuming linearity, superposition principle is applied. The maximum value of $X_{\text {design }}$ is then defined as:

$$
\Delta X_{\text {design }}=\left(\Delta X_{s}\right)_{\text {design }}+\left(\Delta X_{u}\right)_{\text {design }}
$$

Numerically, the RSM were obtained from time domain fully coupled simulations using the DeepLines ${ }^{T M}$ software based on the Finite Element Method [36, 35, 37], therefore accounting for the simultaneous motion of the FPSO and its mooring and riser systems.

\subsection{RSM for the FPSO offset}

The FPSO displacement (or offset) is an important quantity in the design of the mooring system as (i) it is constrained by the maximum permissible excursion of the riser system and (ii) it drives the maximum value of the tension in the mooring lines. The offset is measured relative to the equilibrium position in the abscence of current, wind and waves. The total static offset was defined from the total displacements, in the $X$ and $Y$ directions, including all components due to current, wind and wave, according to:

$$
\begin{gathered}
\Delta X_{\text {tot }}=\Delta X_{U}+\Delta X_{V}+\operatorname{sign}\left(\Delta X_{U}+\Delta X_{V}\right) \cdot \Delta X_{W} \\
\Delta Y_{\text {tot }}=\Delta Y_{U}+\Delta Y_{V}+\operatorname{sign}\left(\Delta Y_{U}+\Delta Y_{V}\right) \cdot \Delta Y_{W}
\end{gathered}
$$

where the subscripts $U, V$ and $W$ refers to the contribution of current, wind and waves, respectively. 

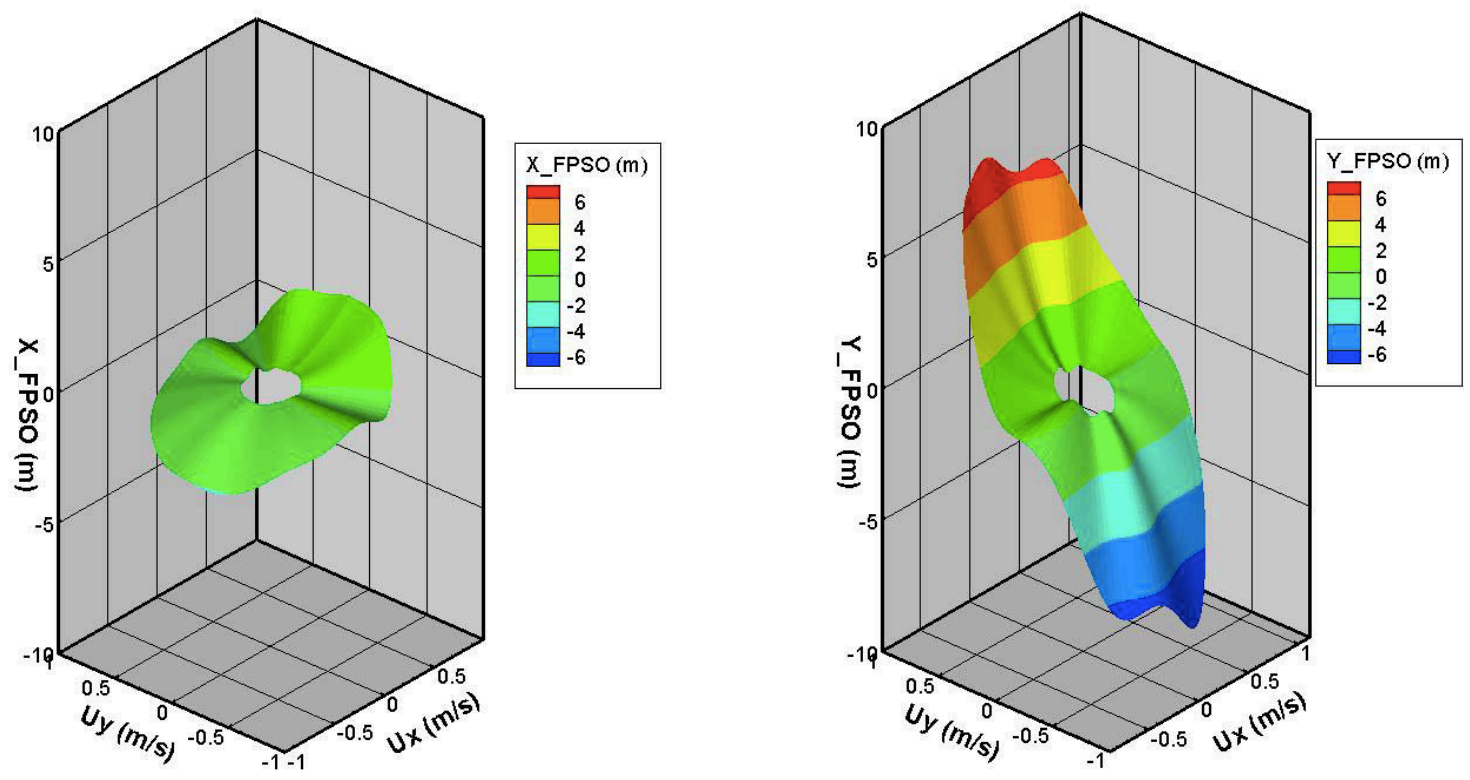

Figure 17: Iso-surface predicted by the RSM for the FPSO displacement as a function $\left(U_{X}, U_{Y}\right)$. Left: $X$ direction $\Delta X\left(U_{X}, U_{Y}\right)$; Right: $Y$ direction $\Delta Y\left(U_{X}, U_{Y}\right)$

\subsubsection{Current-only Response}

The Response Surface Model used to represent the effect of the current on the FPSO displacements in the axial and transverse directions was sought in the form:

$$
\begin{aligned}
\Delta X_{U}\left(\theta_{U}, U\right) & =C_{0 X U}\left(\theta_{U}\right)+C_{1 X U}\left(\theta_{U}\right) \cdot U+C_{2 X U}\left(\theta_{U}\right) \cdot U^{2} \\
\Delta Y_{U}\left(\theta_{U}, U\right) & =C_{0 Y U}\left(\theta_{U}\right)+C_{1 Y U}\left(\theta_{U}\right) \cdot U+C_{2 Y U}\left(\theta_{U}\right) \cdot U^{2}
\end{aligned}
$$

The quadratic dependence in $U$ follows from physical principle of fluid mechanics while the periodicity of the solution with respect to the angular variable $\theta_{U}$ was enforced by representing the coefficients $C_{i X U}$ and $C_{i Y U}$ by means of truncated Fourier series:

$$
C_{i X U}\left(\theta_{U}\right)=\frac{a_{0}^{i}}{2}+\sum_{n=1}^{N} a_{n}^{i} \cdot \cos \left(\frac{\theta_{U}}{2 \pi}\right)
$$

Numerical simulations were performed every 22.5 degrees and Fourier series were truncated after the 8th order. Iso-surface predicted by the RSM for the FPSO displacement in the $X$ and $Y$ directions as a function of the current components $U_{X}=U \cos (\theta)$ and $U_{Y}=U \sin (\theta)$ are plotted in Fig. 17 using the same scales to ease comparison. The displacements due to a current velocity essentially parallel to the $Y$ direction are much larger as the hull area encountered by the flow is larger when the later comes from the side. The current-only offset is then defined as:

$$
R_{U}\left(\theta_{U}, U\right)=\sqrt{\left(\Delta X_{U}\right)^{2}+\left(\Delta Y_{U}\right)^{2}}
$$

and its expected value is given by:

$$
\hat{R}_{U}\left(\theta_{U}, U\right)=R_{U}\left(\theta_{U}, U\right) \cdot p(\operatorname{dir} U, U)
$$

The surface current and associate FPSO offset are plotted in Fig. 18 (left) in the geographic reference frame. The expected value of the current-only offset is plotted in Fig. 18 (right) in the computational reference frame. 

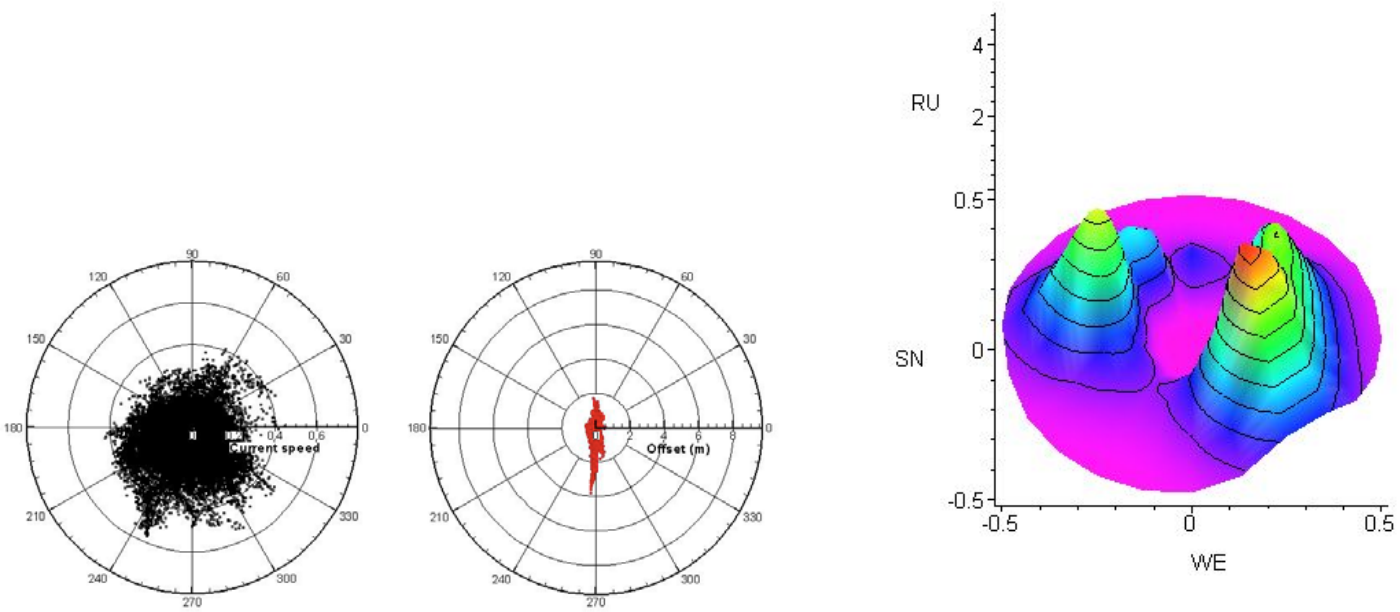

Figure 18: Load and response (FPSO offset) due to current alone as computed on 1 year data used for DSA (Left); Expected value of current-only offset (Right).
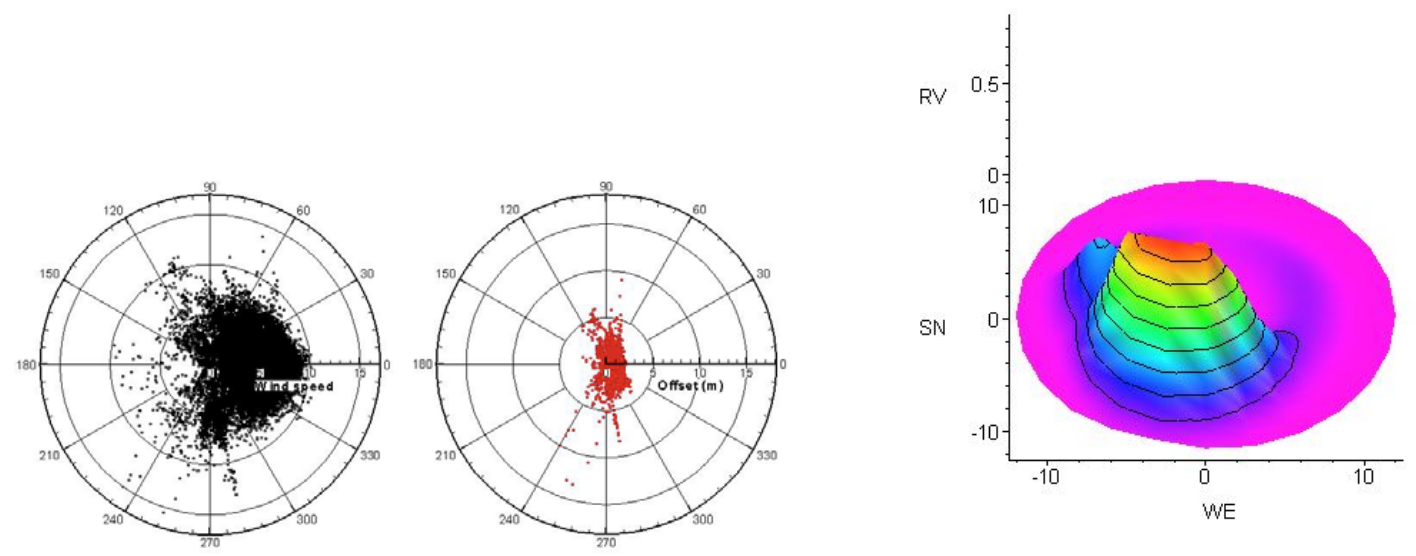

Figure 19: Load and response (FPSO offset) due to wind alone as computed on 1 year data used for DSA (Left); Expected value of wind-only offset (Right).
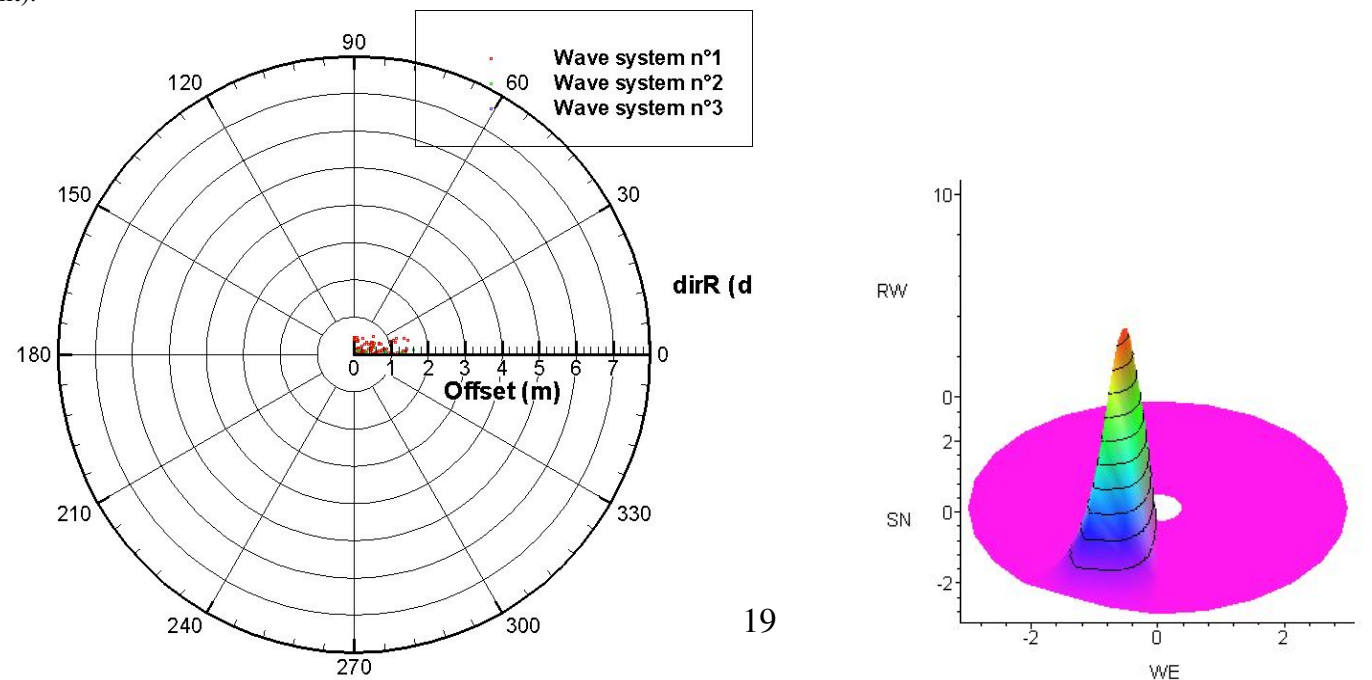

Figure 20: Load and response (FPSO offset) due to wave alone as computed on 1 year data used for DSA (Left); Expected value of wave-only offset (Right). 


\subsubsection{Wind-only Response}

The same procedure was applied to wind-only response. The following relations were used to represent the wind only displacement of the FPSO:

$$
\begin{aligned}
\Delta X_{U}\left(\theta_{V}, V\right) & =C_{0 X V}\left(\theta_{V}\right)+C_{1 X V}\left(\theta_{V}\right) \cdot V+C_{2 X V}\left(\theta_{V}\right) \cdot V^{2} \\
\Delta Y_{U}\left(\theta_{V}, V\right) & =C_{0 Y V}\left(\theta_{V}\right)+C_{1 Y V}\left(\theta_{V}\right) \cdot V+C_{2 Y V}\left(\theta_{V}\right) \cdot V^{2}
\end{aligned}
$$

The wind-only offset is defined by:

$$
R_{V}\left(\theta_{V}, V\right)=\sqrt{\left(\Delta X_{V}\right)^{2}+\left(\Delta Y_{V}\right)^{2}}
$$

and its expected value plotted in Fig. 19 is:

$$
\hat{R}_{V}\left(\theta_{V}, V\right)=R_{V}\left(\theta_{V}, V\right) \cdot p(\operatorname{dir} V, V)
$$

\subsubsection{Wave-only Response}

A Response Surface Model was also constructed to represent the maximum displacement (in a given direction) due to an irregular sea state following a JONSWAP spectra. The model parameters are the significant wave height $H_{s}$, the peak period $T_{p}$, the peak coefficient $\gamma$ and the angular direction $\theta_{W}$. The value of the peak coefficient was considered as a constant $(\gamma=5)$. The displacements $\Delta X_{W}$ and $\Delta Y_{W}$ of the FPSO due to wave only in the axial and transverse directions were sought in the form:

$$
\begin{array}{r}
\Delta X_{W}^{d}\left(\theta_{W}, H_{s}, T_{p}\right)=C_{0 X W}\left(\theta_{W}\right)+C_{1 X W}\left(\theta_{W}\right) \cdot H_{s}+C_{2 X W}\left(\theta_{W}\right) \cdot T_{p} \\
+C_{3 X W}\left(\theta_{W}\right) \cdot H_{s} \cdot T_{p}+C_{4 X W}\left(\theta_{W}\right) \cdot H_{s}^{2}+C_{5 X W}\left(\theta_{W}\right) \cdot T_{p}^{2} \\
\Delta Y_{W}^{d}\left(\theta_{W}, H_{s}, T_{p}\right)=C_{0 Y W}\left(\theta_{W}\right)+C_{1 Y W}\left(\theta_{W}\right) \cdot H_{s}+C_{2 Y W}\left(\theta_{W}\right) \cdot T_{p} \\
+C_{3 Y W}\left(\theta_{W}\right) \cdot H_{s} \cdot T_{p}+C_{4 Y W}\left(\theta_{W}\right) \cdot H_{s}^{2}+C_{5 Y W}\left(\theta_{W}\right) \cdot T_{p}^{2}
\end{array}
$$

The coefficients $C_{i X W}$ and $C_{i Y W}$ were computed for different values of the angular variable $\theta_{W}$ and then represented by means of Fourier series up to the 8 th order. The coefficients were fitted from a series of fully coupled numerical simulations, therefore taking into account first and second order effects, i.e. wave frequency, second order slow drift forces and mooring line dynamics. Only the positive parts from the RSM quadratic approximations were finally retained:

$$
\begin{aligned}
\Delta X_{W}\left(\theta_{W}, H_{s}, T_{p}\right) & =\max \left(\left|\Delta X_{W}^{d}\right|\right) \\
\Delta Y_{W}\left(\theta_{W}, H_{s}, T_{p}\right) & =\max \left(\left|\Delta Y_{W}^{d}\right|\right)
\end{aligned}
$$

The wave-only offset is defined by:

$$
R_{W}\left(\theta_{W}, H_{s}, T_{p}\right)=\sqrt{\left(\Delta X_{W}\right)^{2}+\left(\Delta Y_{W}\right)^{2}}
$$

and its expected value is given by:

$$
\hat{R}_{W}\left(\theta_{W}, H_{s}, T_{p}\right)=R_{W}\left(\theta_{W}, H_{s}, \bar{T}_{p}\right) \cdot p\left(\operatorname{dir} W, H_{s}\right)
$$

with:

$$
\bar{T}_{p}=E\left[T_{p} \mid H_{s}\right]
$$

For given values of the significant height and peak period, the wave response, in $X$ and $Y$ directions, can be evaluated for any wave direction in any direction referring to the East-North frame. In order to visualize the FPSO response sensitivity to wave loadings, the expected value of the wave-only offset, for given values of the wave direction and significant height (mean conditional value for the peak period), has been plotted within the geographic frame (Fig. 20). 


\section{2. $R S M$ for the total tension}

The total tension in one specific mooring line is defined as the combination of the static tension, due to the total static offset, and the dynamic tension due to the waves:

$$
T_{\text {tot }}=T_{\text {static }}\left(\Delta X_{\text {tot }}, \Delta Y_{\text {tot }}\right)+T_{\text {dynamic }}\left(H_{s}, T_{p}, \theta_{W}\right)
$$

\subsubsection{Static Tension}

Variations of the static tension are due to FPSO displacements $(\Delta X, \Delta Y)$ around its equilibrium position. Asymptotically for large displacements the tension in the mooring line is directly proportional to the displacement imposed at the fair-lead of the FPSO. The static tension is related to the total static displacements by means of constant coefficients determined by the RSM analysis:

$$
\begin{array}{r}
T_{\text {static }}\left(\Delta X_{\text {tot }}, \Delta Y_{\text {tot }}\right)=C_{0 T_{s}}+C_{1 T_{s}} \cdot \Delta X_{\text {tot }}+C_{2 T_{s}} \cdot \Delta Y_{\text {tot }} \\
+C_{3 T_{s}} \cdot \Delta X_{\text {tot }} \cdot \Delta Y_{\text {tot }}+C_{4 T_{s}} \cdot \Delta X_{\text {tot }}^{2}+C_{5 T_{s}} \cdot \Delta Y_{\text {tot }}^{2}
\end{array}
$$

\subsubsection{Dynamic Tension}

Following (1), the dynamic tension is defined as twice the value of the dynamic standard deviation of the tension line due to waves:

$$
\begin{array}{r}
\sigma_{T_{d}}\left(H_{s}, T_{p}, \theta_{W}\right)=C_{0 T_{d}}+C_{1 T_{d}} \cdot H_{s}+C_{2 T_{d}} \cdot T_{p} \\
+C_{3 T_{d}} \cdot H_{s} \cdot T_{p}+C_{4 T_{d}} \cdot H_{s}^{2}+C_{5 T_{d}} \cdot T_{p}^{2}
\end{array}
$$

The dynamic standard deviation is provided by the RSM analysis and is represented by a quadratic function of wave variables, i.e. the significant height and the peak period, with directional coefficients. The coefficients involved into the quadratic representation are obviously dependent upon the wave

$$
T_{\text {dynamic }}\left(\theta_{W}, H_{s}, T_{p}\right)=2 \sigma_{T_{d}}\left(\theta_{W}, H_{s}, T_{p}\right)
$$

The coefficients are derived from the RSM analysis and the final mathematical expressions are given in term of Fourier series up to the 8th order. As the initial numerical simulations were performed using a fully coupled model, first and second order effects (wave frequency and low frequency) have been taken into account when deriving these coefficients. Fully coupled numerical simulations of the FPSO motion and of the mooring system were performed for a fixed direction $\theta_{0}$ of the incoming waves. The simulation was launched for 2 hours, always choosing the same seed for wave generation but choosing the origin of time series so as to impose a prescribed value of $H_{\max } / H_{s}$. The simulations were performed with DeepLines ${ }^{T M}$ [37] software, computing the full dynamics of the 16 mooring lines instead of the quasi-static assumption used for the direct numerical simulation presented in Section 3.

\subsection{Accuracy of the Response Surface Model}

Practically, the Response Surface Models have been derived for ballast conditions, corresponding to a FPSO draft of $12 \mathrm{~m}$. This choice was motivated by preliminary results from direct simulation, indicating that the largest offsets were associated with strong wind conditions. Draft distributions (at mid ship and bow) indicate that ballast conditions represent approximately $20 \%$ of the events. As the RSM strongly depends on draft (since the hull surface exposed to current is directly proportional to this quantity), proper comparison between results of RSM and direct simulation should be made for events with similar drafts. Results over the one year long time period considered for which direct simulation was available were therefore filtered with respect to draft, retaining only event with aft draft below $13 \mathrm{~m}$. Results of the comparison are presented in Figures 21. It is observed that the largest offset values are correctly reproduced by the RSM. Some imperfections are present in the RSM for small values of the offset (typically $R<4 m$ ), as was already detected when checking the accuracy of directional distributions. It is believed that reducing the range of allowed variation $\left[0, U_{\max }\right],\left[0, V_{\max }\right],\left[\left(H_{s}\right)_{\min },\left(H_{s}\right)_{\max }\right]$ will improve the accuracy of the RSM for small offsets, should this range be of interest.

The tension in each mooring line has been computed using RSM and successfully compared with results from direct simulation over a period of one year. Fig. 22 shows an example of results for a typical mooring line. The 
dynamic tension varies was computed every three hours with associated $H_{s}$ and $T_{p}$. Though not perfect, the agreement is found to be very satisfactory in view of the level of approximations that have been performed. It should also be noted that error in the offset influence directly the estimation of the mooring lines tension. It should be also mentioned that two completely different software were used: DIODORE [35] for the Direct Simulation Approach (DSA) and DeepLines $^{T M}$ [37] to derive the RSM.

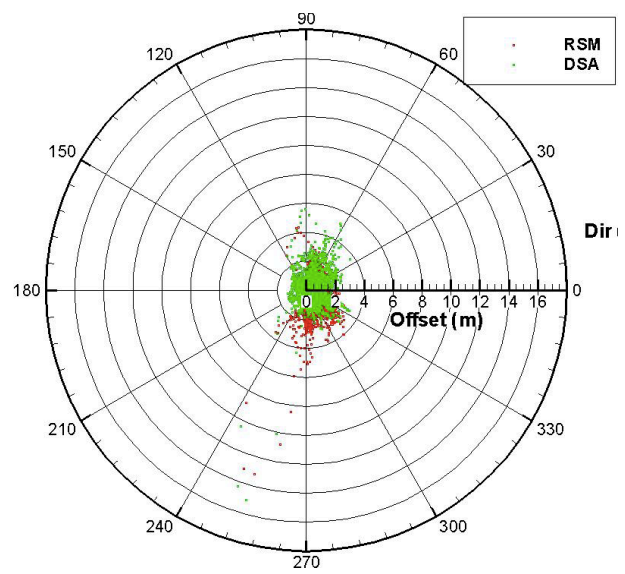

Figure 21: Comparison between results of the Response Surface Model and the direct simulation for the FPSO total offset (due to current, wind and wave) as computed on 1 year data.

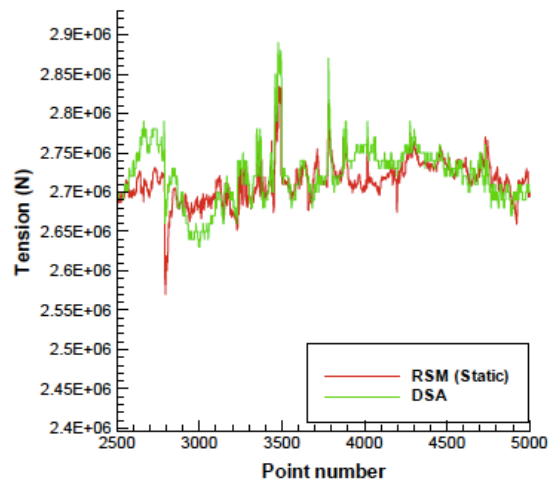

Figure 22: Comparison between results of the Response Surface Model and the direct simulation for the tension in a typical mooring line as computed on 1 year data.

\section{Reliability analysis}

The general framework of structural reliabilty has been described by various authors, see e.g. [60, 61, 62, 63, $64,65,66,67]$ and example of applications of reliability analysis to offshore structures are presented in [68, 69, 70, $71,72,73]$. In the present case, the framework of reliabilty was applied to determine the response and associated environmental conditions that have an associated return period of 100 years or equivalently a $\beta$ index of 4.50 . It has to be noted that, in all configurations, the FPSO is considered in the same ballast condition (with a $12.5 \mathrm{~m}$ draft). The loading condition produces direct and obvious effects upon element loadings and therefore upon response surfaces.

\subsection{Response contours}

Response contours and design points are computed by combining environmental contours and response surfaces in order to derive response maxima. For a given return period $T$, a $\beta$ reliability index, using the Hasofer-Lind definition 
and in association with 3-hour sea states, is derived from the relation

$$
\beta=\Phi^{-1}\left(1-P_{e}\right), \quad \Phi(u)=\frac{1}{\sqrt{2 \pi}} \int_{-i n f t y}^{u} \exp \left(-\frac{t^{2}}{2}\right) d t
$$

where $P_{e}$ is the exceedence probability. In order to determine the design point, defined as the shortest distance between the failure surface and the origin of the standardized variables space, it is useful to reformulate the minimization problem for the limit state function $g(u)$ built from the response function, $h(u)$ for normalized $u$ variables or $H(X)$ for physical $X$ variables, and the (unknown) maximum response:

$$
g(u)=h_{\max }-h(u)
$$

Using the I-FORM formulation, a numerical method was developed in order to solve the optimization problem for the determination of response maxima and design points. An iterative gradient-based method was designed to produce a robust convergence even for complex response functions.

Four different configurations have been studied by combining two environment models and two response functions:

- Environment models

- E0: direction-independent current and wind velocities

- E1: direction-dependant current and wind velocities

- Response functions

- R1: total static offset

- R2: total tension in one specific line

Table 4 summarise the design points coordinates in physical variables and maximum responses at design points that were obtained in the four configurations.

Table 4: Environmental variables and maximum responses at design points

\begin{tabular}{lccccc}
\hline Model & & E0-R1 & E1-R1 & E0-R2 & E1-R2 \\
\hline Physical variables & Units & & & & \\
$\operatorname{dir} U$ & (degrees) & 98 & 138 & 136 & 137 \\
$U$ & $(\mathrm{~m} / \mathrm{s})$ & 0.83 & 1.09 & 0.73 & 1.07 \\
$\operatorname{dir} V$ & $($ degrees) & 127 & 173 & 76 & 165 \\
$V$ & $(\mathrm{~m} / \mathrm{s})$ & 7.89 & 6.56 & 9.11 & 5.99 \\
$\operatorname{dir} W$ & $($ degrees $)$ & 204 & 204 & 203 & 204 \\
$H_{s}$ & $\mathrm{~m})$ & 1.25 & 1.25 & 1.73 & 1.50 \\
$T_{p}$ & $(\mathrm{~s})$ & 12.8 & 12.8 & 13.1 & 13.0 \\
Total offset $R_{\text {tot }}$ & $(\mathrm{m})$ & 11.02 & 12.94 & 9.11 & 12.69 \\
Total tension $T_{\text {tot }}$ & $(\mathrm{kN})$ & 2911 & 2931 & 2910 & 2934 \\
\hline
\end{tabular}

\subsection{Sensitivity analyses}

\subsubsection{Sensitivity to environmental models}

A significant difference is clearly present between results obtained with E0 and E1 models. Taking into account the directionality for the current and wind velocities tends to increase the response level by concentrating the element actions into their principal directions where the induced effects become maximal. The total offset is increased by $18 \%$ (from $11 \mathrm{~m}$ to $13 \mathrm{~m}$ ). The effect on the tension is less apparent but the design point has a different nature depending upon the directionality: current and wind driven response in E0-R2 model and current-only induced response in E1-R2 model. 
Table 5: Reduced index for the first $m$ principal variables.

\begin{tabular}{cccccc}
\hline Model & & $\mathrm{E} 0-\mathrm{R} 1$ & $\mathrm{E} 1-\mathrm{R} 1$ & $\mathrm{E} 0-\mathrm{R} 2$ & $\mathrm{E} 1-\mathrm{R} 2$ \\
\hline$m=1$ & $\beta_{1}$ & 4.074 & 4.417 & 3.776 & 4.365 \\
& $\beta_{1} / \beta$ & 0.9057 & 0.9820 & 0.8395 & 0.9707 \\
\hline$m=2$ & $\beta_{2}$ & 4.328 & 4.484 & 4.061 & 4.469 \\
& $\beta_{2} / \beta$ & 0.9622 & 0.9967 & 0.9028 & 0.9935 \\
\hline$m=3$ & $\beta_{3}$ & 4.451 & 4.492 & 4.416 & 4.491 \\
& $\beta_{3} / \beta$ & 0.9895 & 0.9987 & 0.9816 & 0.9982 \\
\hline
\end{tabular}

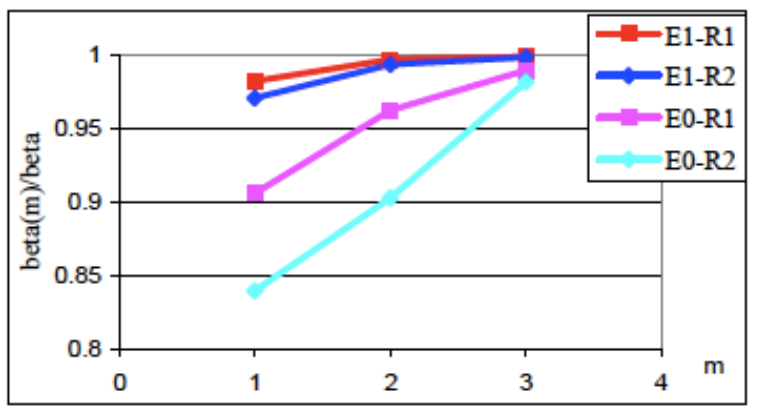

Figure 23: Reliability index comparison for the various models.

At a design point $u^{\star}$, the principal variables $v_{i}$ are defined as the normalized variables reordered in decreasing magnitude. The reduced reliability index $\beta_{m}$ reported in Table 5 were computed with the first $m$ principal variables in order to assess the importance of these variables in the reliability of the model. The directionality, as present in the E1 model, has clearly a significant effect upon the convergence of the reduced index towards the true $\beta$ index as only 2 principal variables are necessary to achieve a greater than .99 convergence for both R1 and R2 response functions (Fig. 23). The current velocity appears to be the most significant parameter since it is associated with the preponderant contribution in all configurations. The wind velocity produces also a significant contribution. The wave parameters are very close to their average values at design points for all models.

\subsubsection{Sensitivity to response functions}

For the R1 and R2 response functions, the results are more coherent between directional E1 models and more dispersed with non-directional E0 models. It is interesting to note the good coherence both in terms of offset and tension for the E1-R1 and E1-R2 configurations: less than $2 \%$ for offset values (12.94 m and $12.69 \mathrm{~m})$ and about $0.1 \%$ for tension values $(2931 \mathrm{kN}$ and $2934 \mathrm{kN})$. The numerical results obtained from different configurations (all in ballast condition with a $12.5 \mathrm{~m}$ draft) indicate that the 100 -year response should be considered to be close to the following values: $12.94 \mathrm{~m}$ for the total offset and $2934 \mathrm{kN}$ for the total tension.

\section{Discussion of results}

Results of the Response Based Design were compared to those of the standard design methodology. Following recommended practice $[2,5]$, classical design consists of running combinations for the environmental loadings with various return periods. For example, the 100 year return period for current can be associated with 1 year return periods for wind and waves. Sensitivity analysis are also required to assess the effect of directionality. After the various combinations have been computed, the worst case is retained and a safety factor is then applied to get the design value of the maximum tension. In the present study, the 100 year return period values of the current, wind and waves were derived from the statistical analysis presented in Section 4.4.1 so that a strict comparison of the design methodology could be made. Results reported in Table 6 indicate that design value predicted using Response Based 


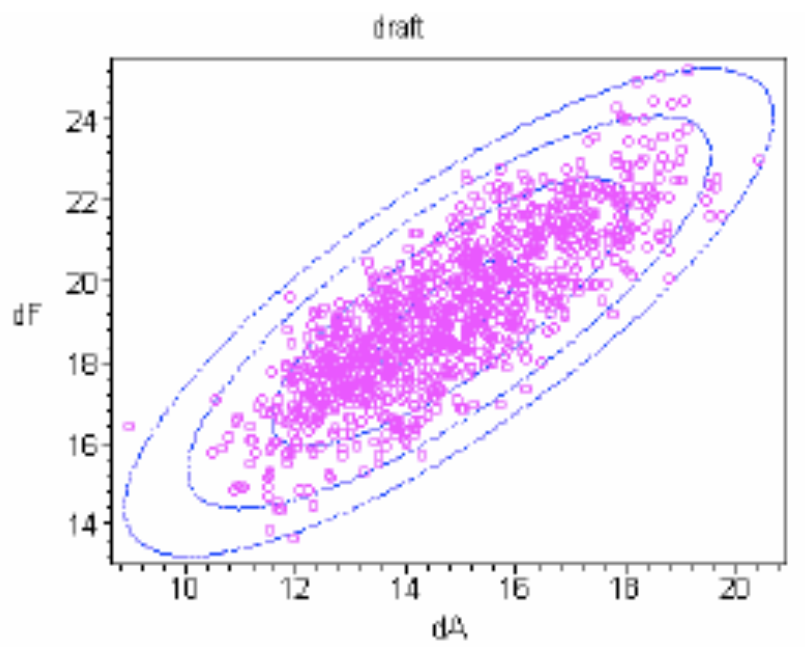

Figure 24: Plot of aft draft (dA) and forward draft $(\mathrm{dF})$ for year 2003

Table 6: Comparison between Response Based Design and conventional approach

\begin{tabular}{lcccc}
\hline Designation & Units & RBD (100y) & $100 \mathrm{y}-1 \mathrm{y}-1 \mathrm{y}$ (Ballast) & $100 \mathrm{y}-1 \mathrm{y}-1 \mathrm{y}$ (D3T2) \\
Offset & $\mathrm{m}$ & 25 & 31 & 29 \\
Max tension L5 & $\mathrm{kN}$ & 3680 & 4170 & 3940 \\
Max tension L14 & $\mathrm{kN}$ & 3629 & 3390 & 4050 \\
\hline
\end{tabular}

Design were roughly $20 \%$ lower than those predicted by the standard approach, in passing providing an estimate of the level of conservatism that is currenty embedded in the design guidance.

Comparison with the results from the reliability analysis was not as straightforward due to the assumption that the FPSO draft corresponds to the ballast loading condition while deriving the Response Surface Models. The choice of the averaged draft was made as the effect of squall events were to be studied at a later stage. To maximise the area exposed to wind gust, it was decided to derive the RSM in the ballast condition. Results of the reliability analysis are therefore valid for the FPSO in the ballast draft condition, while results of Response Based Design are valid for any draft.

In order to assess the validity of the results using I-FORM, a statistical analysis was performed on results from Response Based Design. Direct simulations using the DIODORE software [35] were performed over the year 2003 by means of 24580 computations with 10-mn intervals. The loading conditions used in these simulations were constantly changed on a 3-hour time scale. The aft and forward drafts were also varied as functions of the loading condition as can be seen in Fig. 24. The averaged draft $d$ during the year 2003 could be described as a random variable following a Gaussian distribution with mean value of $17 \mathrm{~m}$ and standard deviation of $1.9 \mathrm{~m}$. In order to estimate the effects of the loading condition upon the FPSO response, the average draft was divided into 3 intervals: Ballast condition $(12 \leq d<15.5 m)$, Intermediate condition $(15.5 \leq d<18.5 m)$ and Fully-loaded condition $(18.5 \leq d<23 m)$. A statistical analysis was performed on each sub-set to estimate extreme values of FPSO offset and mooring line tension on a weekly basis. Different techniques were used such as fitting the cumulative distribution function with Gumbel

Table 7: Comparison between 100-year values

\begin{tabular}{lcc}
\hline Model & Offset(m) & Tension(kN) \\
RBD : 100-year extreme values computed from statistical results & 12.1 & 2918 \\
I-FORM + RSM : 100-year response maxima at design points & 12.94 & 2934 \\
\hline
\end{tabular}




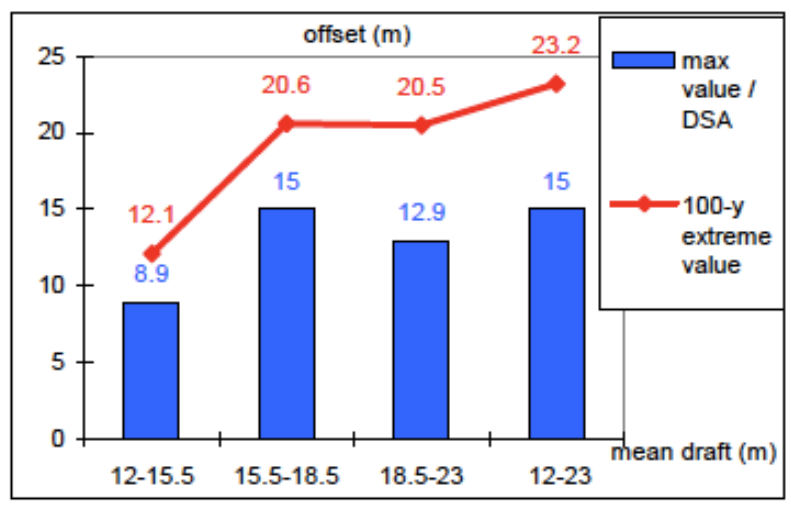

Figure 25: Variations of 100-year extreme values for offset, per mean draft range.

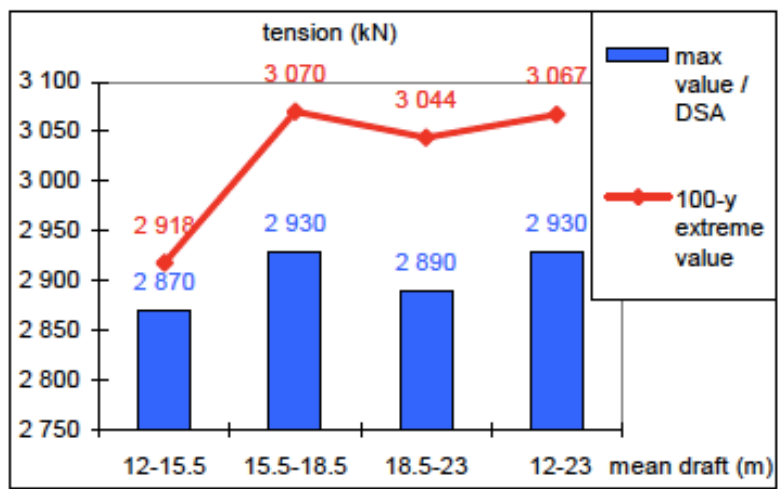

Figure 26: Variations of 100-year extreme values for tension $(\mathrm{kN})$, per mean draft range

and Weibull laws. The average values and standard deviations of the weekly maxima and the 100-year extreme values were computed, as well as the maximum value computed by simulation during the 2003 year. Fig. 25 and 26 show the extreme values for the offset and the mooring tension, respectively. It is clearly apparent that significant variations in response extreme values are due to the loading condition and the associated mean draft, especially in ballast condition compared to the other conditions.

Table 7 present a comparison of the results in Ballast condition. The 100-y extreme values derived from statistical results from RBD compares relatively well with the 100-y maxima obtained by I-FORM+RSM. The reasonably fair agreement between both methods is remarkable taking into account the fact that direct simulation results and RSM coefficients were obtained from different software (DIODORE for RBD and DeepLines ${ }^{T M}$ for RSM). Nevertheless further and more complete validation has to be carried out, in particular for other values of the average draft.

\section{Conclusions}

Concerning the environmental modeling, the example presented in this paper shows the application of a robust methodology to derive joint extremes of met-ocean parameters (wave, wind, current) for use in design of offshore structures. The methodology can be transposed to any offshore location provided that simultaneous records of wave, wind and current are available. Directional PDF were developed for current and wind velocities to take into account their significant directional variations. A modeling based on uniform and Gaussian distributions was used to represent directional PDF and statistical parameters. For the main swell, a direction independent law has been used due to the narrow directional spread. This directional modeling appears as an effective way to deal with directional data for 
I-FORM computations in this particular study because at design points, directions are close to their mean values. The generalization of this approach has to be assessed more precisely.

Through this analysis, the feasibility of the Response Based Design to handle the design of mooring system in West Africa has been established. The advantage of this methodology is the simplicity of its realization but main drawback is the computing time. Note also that the mechanical system was simplified as quasi-static analyses of the mooring line were performed instead of fully coupled simulations to reduce the number of degree of freedom in the model. The present analysis represents 5 days of CPU time on a standard Windows server for more than 25 000 simulations. However, in future project, a good practice should be to try to reduce the number of case by a first screening may be using Response Surface Model as described here to reduce the number of cases to those which will really be used to derived the extreme response. The interest of RBD is also to provide benchmarks so that the quality of low cost simulation method can be assessed. Response surface modeling (RSM) seems to be an appropriate and effective technique to represent the behavior of the FPSO and its mooring/riser systems. Complex response functions, including directional effects, were succesfully represented by RSM. The I-FORM+RSM methodology was applied to the 100-year response of the FPSO in ballast condition, leading to the following key findings:

- The directional effects for current and wind velocities must be included into the environment modeling.

- The current velocity appears to be the preponderant variable for this particular loading condition; also understanding that squall winds were excluded from this study as they would normally create the extreme response of the FPSO in a ballast loading condition.

- In terms of 100-year response, the maximum values obtained at design points by the I-FORM method can be compared with a reasonably fair agreement with extrapolated extreme values from DSA and for the same loading condition (i.e. ballast condition with a mean draft within the $12-15.5 \mathrm{~m}$ range).

This study clearly exhibit possible improvement into design methodology of offshore structures as it puts a milestone toward a rational design based on reliability approach. These results are quite promising but, in order to increase the usability of such a method and its confidence level, further work has to be carried out to carefully assess the validity of the proposed I-FORM + RSM method.

\section{Acknowledgements}

The data used in the study is the joint property of TOTAL E\&P Angola and Esso Exploration Angola (Block 17) Ltd. / BP Exploration (Angola) Ltd. / Statoil Angola Block 17 A.S. / Norsk Hydro Dezassete A.S. / SONANGOL, Sociedade Nacional de Combustveis de Angola, EP. The partners of the study are grateful to them for the authorization to publish this paper.

\section{References}

[1] HSE Report r444 Mooring Integrity.

[2] DNV RP-C301 - DNV Recommended Practice on Mooring Design.

[3] Guidance Note NI 493 DTM R00 E, June 2004, Bureau Veritas.

[4] ISO 19901-7 Petroleum and natural gas industries Specific requirements for offshore structures - Part 7: Station keeping systems for floating offshore structures and mobile offshore units

[5] API RP 2SK - American Petroleum Institute. Recommended Practice for Station Keeping.

[6] Nerzic, R., Frelin, C., Prevosto, M., Quiniou, V. (2007). Joint Distributions of Wind/Waves/Current in West Africa and Derivation of multivariate extreme I-FORM contours, Int Offshore and Polar Eng Conf., ISOPE.

[7] Fontaine, E., Ledoux, A., Leguennec S. (2007), Direct simulation approach for a moored FPSO in West Africa, Int Offshore and Polar Eng Conf., ISOPE, Lisbon.

[8] Orsero, P., Fontaine, E., Quiniou, V. (2007). Reliability and Response Based Design of a Moored FPSO in West Africa, Int Offshore and Polar Eng Conf., ISOPE.

[9] Franois, M., Quiniou, V., Camp, C., Alvarez, J. (2007). Multi-variate I-FORM contours for the design of offshore structures (Practical methodology and application to a West Africa FPSO), Int Offshore and Polar Eng Conf., ISOPE.

[10] Forristall G.Z., CoopeR C.K. Design Current Profiles using Empirical Orthogonal Function (EOF) and Inverse FORM Methods OTC 1997 / $8267 / 1997$

[11] Birades M., Cornell C.A., Ledoigt B. Load Factor Calibration for the Gulf of Guinea - Adaptation of API RP2A-LFRD BOSS Conf / London / 1992 
[12] Cassidy M.J., Houlsby G.T., Taylor E. R. Probabilistic Models Applicable to the Short-Term Extreme Response Analysis of Jack-Up Platforms Journal OMAE / vol $125 / 2003$

[13] Francois M., Giulivo R., Legerstee F. et al Statistics of extreme and fatigue loads in deep water moorings OMAE 2001 Proc. / Rio de Janeiro, Brazil / 2001

[14] Tromans P.S., Vanderschuren L. Response-Based Design of Floaters OGP Workshop / St Albans, England / 2001

[15] Sigurdsson G., Mathisen J., Strom P., Goh T.K. Reliability Reassessment of a Jacket Platform with Gas Seepage in the South China Sea OMAE 2003 / / 2003

[16] Gayton N., Mohamed A., Sorensen J.D. et al Calibration methods for reliability based design codes Structural Safety / 26 (2004) 91-121 / 2004

[17] API RP2T Planning, Designing and Constructing Tension Leg Platforms - Section 4 : Global performance Design and Analysis / / 2004

[18] Kaymaz I., McMahon C.A. A response surface method based on weighted regression for structural reliability analysis Probabilistic Engng Mechanics / 20 (2005) 11-17/2005

[19] Leira B.J., Holmas T., Herfjord K. Application of response surfaces for reliability analysis of marine structures Reliability Engng. \& System Safety / 90 (2005) 131-139 / 2005

[20] Gayton N., Bourinet J.M., Lemaire M. CQ2RS : a new statistical approach to the response surface method for reliability analysis Structural Safety / 25 (2003) 99-121/ 2003

[21] Schuremans L., Van Gemert D. Benefits of splines and neural networks in simulation based structural reliability methods Structural Safety / 27 (2005) 246-261 / 2005

[22] Hagen O., Horte T., Sigurdsson G. Joint Probability of Metocean Phenomena - Applications DNV / report 97-3715 / 1997

[23] Lebas G., Lacasse S., Cornell C.A. Response Surfaces for Reliability Analysis of Jacket Structures OMAE 1992 / vol II $403-409$ / 1992

[24] Rule W.K. A Response Surface for Structural Optimization Journal OMAE / vol 119 / 1997

[25] Campbell B.L., Lawes H.D., Standing R.G.The Use of Response Based Design Methods Applied to Floating Structures FPS Conf / London / 2001

[26] Mazaheri S., Mesbahi E., Downie M.J., Incecik A. Seakeeping Analysis of a Turret-Moored FPSO by using Artificial Neural Networks OMAE 2003 / / 2003

[27] Standing R.G., Thomas D.O., Ahilan R.V. et al The Development of Response-Based Criteria for the Design of FPSOs in Exposed Locations Inst Marine Engineers Conf Innovative Technology

[28] Stiff J.,Ferrari J., Ku A., Spong R. Comparative Risk Analysis of Two FPSO Mooring Configurations OTC / 15377 / 2003

[29] Baar J.J.M., Heyl C.N., Rodenbusch G.Extreme Responses of Turret Moored Tankers OTC 2000 / 12147 / 2000

[30] Ahilan R.V., Standing R.G. Response Based Design for FPSOs - Compromise on Physics or Statistics ? ASRANet / 2002 Colloquium / 2002

[31] Standing R.G., Eichaker R., Lawes H.D. et al Benefits of Applying Response-Based Design Methods to Deepwater FPSOs OTC / 14232 / 2002 for Challenging Environnments / / 1997

[32] Francois M., Quiniou-Ramus V., Legerstee F. et al Directional Metocean Criteria for Mooring and Structural Design of Floating Offshore Structures / JSC147 / 2004

[33] Munier H. Criteres d'environnement multidirectionnels pour l'analyse des structures offshore flottantes BV / rapport ENSTA / 2004

[34] Mazaheri S., Downie M.J. Response Based Method for determining the extreme behaviour of floating offshore platforms Ocean Engng / vol 32 363-693 / 2005

[35] DIODORE (2004) User manual, v3.3.1, PRD Report.

[36] Heurtier, Le Buhan, Fontaine, Le-Cunff, Biolley, 2001, Coupled analysis of moored FPSO with risers. In Proc. 10th Intl Offshore and Polar Engineering Conference, Stavanger, Norway.

[37] DeepLinesTM (2005). Keywords Manual, IFP / PRINCIPIA Report.

[38] Winterstein S.R., Ude T.C., Cornell C.A. et al Environmental Parameters for Extreme Response : Inverse FORM with Omission Factors ICOSSAR-93 / Innsbruck, Austria / 1993

[39] Winterstein, S.R., Ude, T.C., Cornell, C.A., Bjerager, P. and Haver, S. (1993), Environmental Parameters for Extreme Response: Inverse Form with Omission Factors, Proceedings, ICOSSAR-93, Innsbruck, Austria.

[40] Winterstein S.R., Torhaug R., Kumar S. Design Seastates for Extreme Response of Jackup Structures OMAE Conf / vol II 77-84 / 1994

[41] Winterstein S.R., Kumar S., Kleiven G. Environmental Contours and Extreme Response of Deep-Water Floating Structures ASCE Eng. Mech. Spec. Conf. / / 1995

[42] Morton I.D., Bowers Extreme Value Analysis in a Multivariate Offshore Environment J. Applied Ocean Research / 18 (1996) $303-317$ / 1996

[43] Bitner-Gregersen E.M., Hagen O., Mathisen J. Joint Probability of Metocean Phenomena - Models for Voring, Statfjord and Ekofisk DNV / report 97-2034 / 1997

[44] Botelho Machado U. Some Uncertainties of Joint Environmental Modelling DNV / report 97-2033 / 1997

[45] Repko A., Van Gelder P.H., Voortman H.G. et al Bivariate Statistical Analysis of Wave Climate ICCE Proc. / 583-596 / 2000

[46] Guedes Soares C., Pascoal R. Experimental Study of the Probability Distributions of Green Water on the Bow of Floating Production Platforms OMAE 2002 / $28626 / 2002$

[47] Ditlevsen O. Stochastic Model for Joint Wave and Wind Loads on Offshore Structures, Structural Safety / 24 (2002) 139-163 / 2002

[48] State of the Art Review on Wave Description in Current Design Practice and Marine Operations DNV Envir MaxWave / report 2001-1491/ 2001

[49] Haver S. On the Prediction of Extreme Wave Crest Heights 2003

[50] Quiniou-Ramus V., Hoche M.A., Francois M. et al Recent Breakthroughs in the Analysis of Total E\&P Angola Block 17 Wind / Wave / Current Records and their Impact on Floating Structures Design Deep Offshore Technology Conf. / Marseilles, France / 2003

[51] Kleiven G., Haver S. Met-ocean Contour Lines for Design : Correction for Omitted Variability in the Response Process ISOPE 2004 / Toulon, France / 2004

[52] Repko A., Van Gelder P.H.A.J.M., Voortman H.G., Vrijling J.K. Bivariate description of offshore wave conditions with physics-based extreme value statistics Applied Ocean Research / 26 (2004) 162-170 / 2004 
[53] Haver S., Kleiven G. Environmental Contour Lines for Design Purposes - Why and When ? OMAE 2004 / Vancouver, Canada / 2004

[54] Bhattacharya B., Wang S., Basu R. et al. Reliability-Based Combination of Environmental Parameters for the Design of Novel Floating Structures OMAE / 1999 Conference / 1999

[55] Lihong, Foschi R.O. An inverse reliability method and its application Structural Safety / 20 (1998) 257-270/ 1998

[56] Niedzwecki J.M., van de lindt J.W., Yao J.T.P. Estimating Extreme Tendon Response using Environmental Contours Engineering Structures / $207601-607$ / 1998

[57] Saranyasoontom K., Manuel L. Efficient Models for Wind Turbine Extreme Loads using Inverse Reliability Wind Engng \& Indust Aerodynamics / 92 (2004) 789-804 / 2004

[58] Vandelindt J.W., Niedzwecki J.M. Environmental Contour Analysis in Earthquake Engineering Engineering Structures / 22 (2000) 1661 -1676 / 2000

[59] Rosenblatt, M. Remarks on a Multivariate Transformation. Ann. Math. Statist. Vol. 23, No. 3 (1952), 470-472.

[60] Ditlevsen O., Madsen H.O.Structural Reliability Methods TU Denmark / Dept. Mech. Engng. / 2005

[61] Faber M.H. Methods of Structural Reliability Theory - An Introduction Swiss Federal Institute Technology // 2001

[62] Koutsourelakis P.S., Pradlwarter H.J., Schueller G.I. Reliability of structures in high dimensions, part I : algorithms and applications Probabilistic Engng. Mechanics / 19 (2004) 409-417 / 2004

[63] Lemaire M. Fiabilite des structures mecaniques - Couplage mecano-fiabiliste statique IFMA LaRAMA / / 2002

[64] Procaccia H., Morilhat P. Fiabilite des structures des installations industrielles Coll. DER/EDF 94 / Eyrolles / 1996

[65] Raizer V. Theory of Reliability in Structural Design ASME Appl. Mech. Review / vol 57 n1 / 2004

[66] Schueller G.I., Pradlwarter H.J., Koutsourelakis P.S. A comparative study of reliability estimation procedures for high dimensions ASCE Engng Mech. Conf; / Seattle, 2003 / 2003

[67] Thoft-Christensen P., BAKER M.J. Structural Reliability Theory and Its Applications Springer Verlag // 1982

[68] Manuel L., Winterstein S.R. Reliability-Based Predictions of a Design Air Gap for Floating Offshore Structures, ASCE Conf Proba Mech Struct Reliab / PMC2000-343 / 2000

[69] Ronold K.O., Larsen G.C. Reliability Based Design of Wind Turbine Rotor Blades against Failure in Ultimate Loading Engng Structures / vol $22565-574 / 2000$

[70] Winterstein S.R., Kumar S. Reliability of Floating Structures : Extreme Response and Load Factor Design OTC / 7758 / 1995

[71] Winterstein S.R., Engebretsen K. Reliability -Based Prediction of Design Loads and Responses for Floating Ocean Structures OMAE 1998 / / 1998

[72] Winterstein S.R., Jha A.K., Kumar S. J. Reliability of Floating Structures : Extreme Response and Load Factor Design Waterway, Port, Coastal and Ocean Engng / july/august 1999 / 163-169 / 1999

[73] Manuel L., Schmucker D.G., Cornell C.A., Carballo J.E. A reliability-based design format for jacket platforms under wave loads Marine Structures / 11 (1998) 413-428 / 1998 